\title{
Fundamental solution of the multi-dimensional time fractional telegraph equation*
}

\author{
M. Ferreira ${ }^{\dagger, \ddagger} \quad$ M.M. Rodrigues ${ }^{\ddagger} \quad$ N. Vieira ${ }^{\ddagger}$ \\ $\dagger$ School of Technology and Management \\ Polytechnic Institute of Leiria \\ P-2411-901, Leiria, Portugal. \\ $\ddagger$ CIDMA - Center for Research and Development in Mathematics and Applications \\ Department of Mathematics, University of Aveiro \\ Campus Universitário de Santiago, 3810-193 Aveiro, Portugal.
}

E-mails: milton.ferreira@ipleiria.pt,mferreira@ua.pt,mrodrigues@ua.pt,nloureirovieira@gmail.com

\begin{abstract}
In this paper we study the fundamental solution (FS) of the multidimensional time-fractional telegraph equation with time-fractional derivatives of orders $\alpha \in] 0,1]$ and $\beta \in] 1,2]$ in the Caputo sense. Using the Fourier transform we obtain an integral representation of the FS expressed in terms of a multivariate MittagLeffler function in the Fourier domain. The Fourier inversion leads to a double Mellin-Barnes type integral representation and consequently to a $\mathrm{H}$-function of two variables. An explicit series representation of the FS, depending on the parity of the dimension, is also obtained. As an application, we study a telegraph process with Brownian time. Finally, we present some moments of integer order of the FS, and some plots of the FS for some particular values of the dimension $n$ and of the fractional parameters $\alpha$ and $\beta$.
\end{abstract}

Keywords: Time-fractional telegraph operator; Fundamental solutions; Caputo fractional derivative; Multivariate Mittag-Leffler functions; H-function of two variables; Double Mellin-Barnes type integrals.

MSC 2010: 35R11, 26A33, 35A08, 35A22, 35C15, 60G22.

\section{Introduction}

In the last years, the interest in the study of fractional differential equations has increased considerably due essentially to the wide range of applications. These type of equations are used mostly to model processes in the fields of engineering, viscoelastic materials, hydrology, system control, just to mention some (see [11, 17]). One important type of fractional differential equations is the class of space-time fractional diffusion equations, which models anomalous diffusion and wave phenomena. Recently, two of the authors studied the multidimensional time-fractional diffusion-wave equation (see [8]). This equation is a particular case of the time-fractional telegraph equation. In the one-dimensional case the telegraph equation is given by

$$
\partial_{t t}^{2} u(x, t)+a \partial_{t} u(x, t)+b u(x, t)=c^{2} \partial_{x x}^{2} u(x, t),
$$

where $x \in \mathbb{R}, t>0, a, b, c \in \mathbb{R}, c>0$. In contrast to the wave equation, the telegraph equation has the potential to describe both diffusive and wave-like phenomena, due to the simultaneous presence of first and second order time derivatives. Telegraph equations have an extraordinary importance in electrodynamics (the scalar Maxwell equations are of this type), in the theory of damped vibrations and in probability because they are

\footnotetext{
*The final version is published in Fractional Calculus and Applied Analysis, 20-No.4, (2017), 868-894. It as available via the website https://doi.org/10.1515/fca-2017-0046
} 
connected with finite velocity random motions. In this paper we study the following class of multidimensional time-fractional telegraph equation

$$
D_{t}^{\beta} u(x, t)+a D_{t}^{\alpha} u(x, t)=c^{2} \Delta_{x} u(x, t),
$$

where $x \in \mathbb{R}^{n}, t>0,1<\beta \leq 2,0<\alpha \leq 1, a \geq 0, c>0$, and $\Delta_{x}$ is the Laplace operator in $\mathbb{R}^{n}$. For $\gamma>0, D_{t}^{\gamma}$ is the Caputo fractional derivative of order $\gamma$ defined by

$$
D_{t}^{\gamma} u(t)=\left\{\begin{array}{cl}
\frac{1}{\Gamma(m-\gamma)} \int_{0}^{t}(t-s)^{-\gamma+m-1} u^{(m)}(s) d s, & m-1<\gamma<m \\
u^{(m)}(t), & \gamma=m
\end{array}\right.
$$

where $u^{(m)}:=\frac{d^{m} u}{d t^{m}}, m \in \mathbb{N}$.

The use of fractional derivatives in the telegraph equation give us the possibility of describing memory and heredity properties of telegraph processes (see $[11,17]$ ). One of the first works studying the time-fractional telegraph equation is the paper of Cascaval et al. (see [5]). Here, the authors discussed some properties of the time-fractional telegraph equation in $\mathbb{R} \times \mathbb{R}^{+}$such as the well-posedness and the asymptotic behavior of the solutions, by using the Riemann-Liouville approach. In [16], Orsingher and Beghin obtained the FS of the timefractional telegraph equation of order $2 \alpha$ in $\mathbb{R} \times \mathbb{R}^{+}$and gave a representation of their inverses in terms of stable densities. For the special case $\alpha=\frac{1}{2}$, the authors showed that the FS is the probability density of a telegraph process with Brownian time. In [4] it was discussed the solution of a general space-time fractional telegraph equation by means of the Laplace and Fourier transforms in the variables $x \in \mathbb{R}$ and $t \in \mathbb{R}^{+}$, respectively. In [19] it was obtained the solutions of the space-time fractional telegraph equation in $\mathbb{R} \times \mathbb{R}^{+}$in terms of Mittag-Leffler functions, using an operational approach. In [15], Mamchuev considered the inhomogeneous time-fractional telegraph equation with Caputo derivatives, and obtained a general representation of regular solution in rectangular domain in terms of fundamental solution and appropriate Green functions. Regarding the multidimensional case, in [6] the authors discussed and derived the solution of the time-fractional telegraph equation in $\mathbb{R}^{n} \times \mathbb{R}^{+}$with three kinds of nonhomogeneous boundary conditions, by the method of separation of variables.

The aim of this paper is to obtain an explicit integral and series representations for the FS of the timefractional telegraph equation in an arbitrary dimension. A representation of the FS in the form of an absolute convergent series enables to handle these functions in an easier way and to apply them to study other Cauchy problems. Our results generalize those presented in [8] for the case of the multidimensional time-fractional diffusion-wave equation.

The structure of the papers reads as follows: in the preliminaries section we recall some basic concepts about fractional calculus, special functions and integral transforms. In Section 3 we construct integral and series representations for the FS of the time-fractional telegraph operator in $\mathbb{R}^{n} \times \mathbb{R}^{+}$. More precisely, we apply operational techniques via the Fourier transform obtaining an integral representation of the FS expressed in terms of a multivariate Mittag-Leffler function. After Fourier inversion, the FS is represented as a double Mellin-Barnes type integral and, consequently, as a H-function of two variables. For the explicit calculation of the double Mellin-Barnes type integral we apply the Residue Theorem obtaining an explicit double series representation of the FS, which depends on the parity of the dimension. In Section 4 we deduce a general expression for some moments of integer order of the FS. In Section 5 we present an application of our results to the law of a telegraph process with Brownian time. Finally, in Section 6 we present and discuss some plots of the FS obtained in Section 3 for some particular values of the dimension $n$ and of the fractional parameters $\alpha$ and $\beta$. 


\section{Special functions and basic results}

In this section we present the main tools concerning fractional derivatives and special functions that we will use in our analysis. We start by recalling some properties of the Gamma function that are used in this paper.

$$
\begin{aligned}
& \Gamma(z+n)=(z)_{n} \Gamma(z), \quad n \in \mathbb{N} \\
& \Gamma(z-n)=\frac{(-1)^{n} \Gamma(z)}{(1-z)_{n}}, \quad n \in \mathbb{N} \\
& \Gamma\left(z+\frac{1}{2}\right)=\frac{2^{1-2 z} \sqrt{\pi} \Gamma(2 z)}{\Gamma(z)} \\
& \Gamma(z) \Gamma(-z)=\frac{-\pi}{z \sin (\pi z)} \\
& \Gamma\left(\frac{1}{2}-z\right)=\frac{\pi}{\cos (\pi z) \Gamma\left(\frac{1}{2}+z\right)} .
\end{aligned}
$$

For the poles of the Gamma function we have the following relation:

$$
\operatorname{res}_{s=-k} \Gamma(s)=\frac{(-1)^{k}}{k !}, \quad k \in \mathbb{Z}_{0}^{+} .
$$

A special function that appears when we solve our fractional differential equation is the multivariate MittagLeffler function.

Definition 2.1 (see [13]) The multivariate Mittag-Leffler function $E_{\left(a_{1}, \ldots, a_{n}\right), b}\left(z_{1}, \ldots, z_{n}\right)$ of $n$ complex variables $z_{1}, \ldots, z_{n} \in \mathbb{C}$ with complex parameters $a_{1}, \ldots, a_{n} \in \mathbb{C}$ (with positive real parts) is defined by

$$
E_{\left(a_{1}, \ldots, a_{n}\right), b}\left(z_{1}, \ldots, z_{n}\right)=\sum_{k=0}^{+\infty} \sum_{\substack{l_{1}+\ldots+l_{n}=k \\
l_{1}, \ldots, l_{n} \geq 0}}\left(\begin{array}{c}
k \\
l_{1}, \ldots, l_{n}
\end{array}\right) \frac{\prod_{i=1}^{n} z_{i}^{l_{i}}}{\Gamma\left(b+\sum_{i=1}^{n} a_{i} l_{i}\right)}
$$

where the multinomial coefficients are given by

$$
\left(\begin{array}{c}
k \\
l_{1}, \ldots, l_{n}
\end{array}\right):=\frac{k !}{l_{1} ! \times \ldots \times l_{n} !}
$$

In particular, when $n=2$, the multivariate Mittag-Leffler function (9) can be written as

$$
\begin{aligned}
E_{\left(a_{1}, a_{2}\right), b}\left(z_{1}, z_{2}\right) & =\sum_{k=0}^{+\infty} \sum_{\substack{l_{1}+l_{2}=k \\
l_{1}, l_{2} \geq 0}} \frac{k !}{l_{1} ! l_{2} !} \frac{z_{1}^{l_{1}} z_{2}^{l_{2}}}{\Gamma\left(b+a_{1} l_{1}+a_{2} l_{2}\right)} \\
& =\sum_{l_{1}=0}^{+\infty} \sum_{l_{2}=0}^{+\infty} \frac{\left(l_{1}+l_{2}\right) !}{l_{1} ! l_{2} !} \frac{z_{1}^{l_{1}} z_{2}^{l_{2}}}{\Gamma\left(b+a_{1} l_{1}+a_{2} l_{2}\right)} .
\end{aligned}
$$

For $n=1$, the multivariate Mittag-Leffler function (9) reduces to the two-parameter Mittag-Leffler function

$$
E_{a_{1}, b}\left(z_{1}\right)=\sum_{k=0}^{\infty} \frac{z_{1}^{k}}{\Gamma\left(b+k a_{1}\right)}, \quad a_{1}, b, z_{1} \in \mathbb{C} ; \Re\left(a_{1}\right), \Re(b)>0 .
$$

For general properties of the Mittag-Leffler function see $[10,13]$.

Definition 2.2 (see [12]) The Fox H-function $H_{p, q}^{m, n}$ is defined via a Mellin-Barnes type integral of the form

$$
H_{p, q}^{m, n}\left[z \mid \begin{array}{c}
\left(a_{1}, \alpha_{1}\right), \ldots,\left(a_{p}, \alpha_{p}\right) \\
\left(b_{1}, \beta_{1}\right), \ldots,\left(b_{q}, \beta_{q}\right)
\end{array}\right]=\frac{1}{2 \pi i} \int_{\mathcal{L}} \frac{\prod_{j=1}^{m} \Gamma\left(b_{j}+\beta_{j} s\right) \prod_{i=1}^{n} \Gamma\left(1-a_{i}-\alpha_{i} s\right)}{\prod_{i=n+1}^{p} \Gamma\left(a_{i}+\alpha_{i} s\right) \prod_{j=m+1}^{q} \Gamma\left(1-b_{j}-\beta_{j} s\right)} z^{-s} d s
$$

where $m, n, p, q \in \mathbb{N}$ such that $0 \leq m \leq q, 0 \leq n \leq p, a_{i}, b_{j} \in \mathbb{C}$, and $\alpha_{i}, \beta_{j} \in \mathbb{R}^{+}(i=1,2, \ldots, p ; j=$ $1,2, \ldots, q)$. 
The conditions for the existence of the Fox H-function and the orientation of the contour $\mathcal{L}$ are given by Theorem 1.1 in [12]. More general, we have the H-function of $r$ complex variables. Here, we present only the definition of the H-function of two complex variables.

Definition 2.3 (see [3]) The H-function of two complex variables is defined via a Mellin-Barnes type integral of the form

$$
\begin{array}{r}
H[x, y]=H_{p_{1}, q_{1} ; p_{2}, q_{2} ; p_{3}, q_{3}}^{0, n_{1} ; m_{2}, n_{2} ; m_{3}, n_{3}}\left[\begin{array}{r|r}
x & \left(a_{j} ; \alpha_{j}, A_{j}\right)_{1, p_{1}} ;\left(c_{j}, \gamma_{j}\right)_{1, p_{2}} ;\left(e_{j}, E_{j}\right)_{1, p_{3}} \\
y & \left(b_{j} ; \beta_{j}, B_{j}\right)_{1, q_{1}} ;\left(d_{j}, \delta_{j}\right)_{1, q_{2}} ;\left(f_{j}, F_{j}\right)_{1, q_{3}}
\end{array}\right] \\
=\frac{1}{(2 \pi i)^{2}} \int_{\mathcal{L}_{2}} \int_{\mathcal{L}_{1}} \phi(s, t) \phi_{1}(s) \phi_{2}(w) x^{s} y^{w} d s d w
\end{array}
$$

where

$$
\begin{aligned}
& \phi(s, w)=\frac{\prod_{i=1}^{n_{1}} \Gamma\left(1-a_{i}+\alpha_{i} s+A_{i} w\right)}{\prod_{i=n_{1}+1}^{p_{1}} \Gamma\left(a_{i}-\alpha_{i} s-A_{i} w\right) \prod_{j=1}^{q_{1}} \Gamma\left(1-b_{j}+\beta_{j} s+B_{j} w\right)}, \\
& \phi_{1}(s)=\frac{\prod_{j=1}^{m_{2}} \Gamma\left(d_{j}-\delta_{j} s\right) \prod_{i=1}^{n_{2}} \Gamma\left(1-c_{i}+\gamma_{i} s\right)}{\prod_{j=m_{2}+1}^{q_{2}} \Gamma\left(1-d_{j}+\delta_{j} s\right) \prod_{i=n_{2}+1}^{p_{2}} \Gamma\left(c_{i}-\gamma_{i} s\right)}, \\
& \phi_{2}(w)=\frac{\prod_{j=1}^{m_{3}} \Gamma\left(f_{j}-F_{j} w\right) \prod_{i=1}^{n_{3}} \Gamma\left(1-e_{i}+E_{i} w\right)}{\prod_{j=m_{3}+1}^{q_{3}} \Gamma\left(1-f_{j}+F_{j} w\right) \prod_{i=n_{3}+1}^{p_{3}} \Gamma\left(e_{i}-E_{i} w\right)},
\end{aligned}
$$

with $x, y \in \mathbb{C}, m_{i}, n_{i}, p_{i}, q_{i} \in \mathbb{Z}$ such that $0 \leq m_{i} \leq q_{i}, 0 \leq n_{i} \leq p_{i}(i=1,2,3), a_{i}, b_{j}, c_{i}, d_{j}, e_{i}, f_{j} \in \mathbb{C}$, and $\alpha_{i}, A_{i}, \beta_{j}, B_{j}, \gamma_{i}, \delta_{j}, E_{i}, F_{j} \in \mathbb{R}^{+}$.

The conditions for the analyticity and convergence of this special function, its general properties, and the orientation of the contours $\mathcal{L}_{1}$ and $\mathcal{L}_{2}$ are studied e.g. in [3]. Moreover, when all poles of the integrand are simple, we have the following asymptotic behaviour for large values of $x$ and $y$ (see [2])

$$
H[x, y]=O\left(|x|^{\beta_{1}},|y|^{\beta_{2}}\right)
$$

where

$$
\begin{array}{ll}
\beta_{1}=\max \left\{\Re\left(\frac{c_{j}-1}{\delta_{j}}\right)\right\}, & j=1,2, \ldots, n_{2}, \\
\beta_{2}=\max \left\{\Re\left(\frac{e_{j}-1}{E_{j}}\right)\right\}, & j=1,2, \ldots, n_{3} .
\end{array}
$$

Now we recall the definition of the spaces $C_{\alpha}, \alpha \in \mathbb{R}$, and $C_{\alpha}^{m}, m \in \mathbb{N}$ given in [13].

Definition 2.4 (see [13]) A real or complex-valued function $f(t), t>0$, is said to be in the space $C_{\alpha}, \alpha \in \mathbb{R}$, if there exists a real number $p>\alpha$ such that $f(t)=t^{p} f_{1}(t)$ for some function $f_{1} \in C[0, \infty)$.

It is easy to see that $C_{\alpha}$ is a vector space and the set of spaces $C_{\alpha}$ is ordered by inclusion according to $C_{\alpha} \subseteq C_{\beta}$ if and only if $\alpha \geq \beta$.

Definition 2.5 (see [13]) A function $f(t), t>0$, is said to be in the space $C_{\alpha}^{m}, m \in \mathbb{N}$, if and only if $f^{(m)} \in C_{\alpha}$.

The next theorem will be used in our analysis and allow us to solve general linear differential equations with constant coefficients and Caputo derivatives.

Theorem 2.6 (see [13, Thm 4.1]) Let $\mu>\mu_{1}>\ldots>\mu_{n} \geq 0, m_{i}-1<\mu_{i} \leq m_{i}, m_{i} \in \mathbb{N}, \lambda_{j} \in \mathbb{R}, i=1, \ldots, n$. Consider the initial value problem

$$
\left\{\begin{array}{l}
D_{t}^{\mu} y(t)-\sum_{i=1}^{n} \lambda_{i} D_{t}^{\mu_{i}} y(t)=g(t) \\
y^{(k)}(0)=c_{k} \in \mathbb{R}, \quad k=0, \ldots, m-1, m-1<\mu \leq m,
\end{array}\right.
$$


where $g$ is assumed to lie in $C_{-1}$ if $\mu \in \mathbb{N}_{0}$ or in $C_{-1}^{1}$ if $\mu \notin \mathbb{N}_{0}$. Then (14) has a unique solution in the space $C_{-1}^{m}$ of the form

$$
y(t)=\int_{0}^{t} s^{\mu-1} E_{\left(\mu-\mu_{1}, \ldots, \mu-\mu_{n}\right), \mu}\left(\lambda_{1} s^{\mu-\mu_{1}}, \ldots, \lambda_{1} s^{\mu-\mu_{n}}\right) g(t-s) d s+\sum_{k=0}^{m-1} c_{k} u_{k}(t), \quad t \geq 0,
$$

with

$$
u_{k}(t)=\frac{t^{k}}{k !}+\sum_{i=l_{k}+1}^{n} \lambda_{i} t^{k+\mu-\mu_{i}} E_{\left(\mu-\mu_{1}, \ldots, \mu-\mu_{n}\right), k+1+\mu-\mu_{i}}\left(\lambda_{1} t^{\mu-\mu_{1}}, \ldots, \lambda_{n} t^{\mu-\mu_{n}}\right), \quad k=0, \ldots, m-1 .
$$

The natural numbers $l_{k}, k=0, \ldots, m-1$ are determined from the conditions $m_{l_{k}} \geq k+1$ and $m_{l_{k}+1} \leq k$. In the case $m_{i} \leq k, i=0, \ldots, m-1$, we set $l_{k}:=0$, and of $m_{i} \geq k+1, i=0, \ldots, m-1$, then $l_{k}:=n$.

\section{Fundamental solution of the multidimensional time fractional tele- graph equation}

In this section we compute the FS for the multidimensional time-fractional telegraph equation, i.e., we look for the function $G_{n}^{\alpha, \beta}(x, t)$ that satisfies the following Cauchy problem:

$$
\left\{\begin{array}{l}
\left(D_{t}^{\beta}+a D_{t}^{\alpha}-c^{2} \Delta_{x}\right) G_{n}^{\alpha, \beta}(x, t)=0 \\
G_{n}^{\alpha, \beta}(x, 0)=\delta(x) \\
\frac{\partial G_{n}^{\alpha, \beta}}{\partial t}(x, 0)=0
\end{array}\right.
$$

where $x \in \mathbb{R}^{n}$ and $\delta$ is the delta Dirac function in $\mathbb{R}^{n}$. Applying the Fourier transform in $\mathbb{R}^{n}$ to (15) we get the following initial-value problem:

$$
\left\{\begin{array}{l}
\left(D_{t}^{\beta}+a D_{t}^{\alpha}+c^{2}|k|^{2}\right) \widehat{G}_{n}^{\alpha, \beta}(\kappa, t)=0 \\
\widehat{G}_{n}^{\alpha, \beta}(\kappa, 0)=1 \\
\frac{\partial \widehat{G}_{n}^{\alpha, \beta}}{\partial t}(\kappa, 0)=0
\end{array} .\right.
$$

To solve the problem (16) we apply Theorem 2.6 with $\lambda_{1}=-a, \lambda_{2}=-c^{2}|\kappa|^{2}, \mu=\beta, \mu_{1}=\alpha, \mu_{2}=0, n=2$, $m=2, g(t)=0, k=0$, yielding the following solution:

$$
\widehat{G}_{n}^{\alpha, \beta}(\kappa, t)=1-c^{2}|\kappa|^{2} t^{\beta} E_{(\beta-\alpha, \beta), 1+\beta}\left(-a t^{\beta-\alpha},-c^{2}|\kappa|^{2} t^{\beta}\right) .
$$

By (11) we can write the multivariate Mittag-Leffler function that appears in (17) in the form of a double series:

$$
E_{(\beta-\alpha, \beta), 1+\beta}\left(-a t^{\beta-\alpha},-c^{2}|\kappa|^{2} t^{\beta}\right)=\sum_{j=0}^{+\infty} \sum_{k=0}^{+\infty} \frac{\Gamma(1+k+j)}{\Gamma(1+\beta+(\beta-\alpha) k+\beta j) k ! j !}\left(-a t^{\beta-\alpha}\right)^{k}\left(-c^{2}|\kappa|^{2} t^{\beta}\right)^{j} .
$$

Moreover, we have the following lemma.

Lemma 3.1 The multivariate Mittag-Leffler function in (17) has the following representation in the form of double Mellin-Barnes integral

$E_{(\beta-\alpha, \beta), 1+\beta}\left(-a t^{\beta-\alpha},-c^{2}|\kappa|^{2} t^{\beta}\right)=\frac{1}{(2 \pi i)^{2}} \int_{\mathcal{L}_{1}} \int_{\mathcal{L}_{2}} \frac{\Gamma(1+s+w) \Gamma(-s) \Gamma(-w)}{\Gamma(1+\beta+(\beta-\alpha) w+\beta s)}\left(c^{2}|\kappa|^{2} t^{\beta}\right)^{s}\left(a t^{\beta-\alpha}\right)^{w} d w d s$.

Proof: Considering the change of variables $s \mapsto-s$ and $w \mapsto-w$ in the right hand side of (19) and taking into account the theory of the Fox H-function presented in [12] we have that the integral with respect to the variable $s$ is convergent and is equal to

$$
\underbrace{\frac{1}{2 \pi i} \int_{\mathcal{L}_{1}} \frac{\Gamma(1-s-w) \Gamma(s)}{\Gamma(1+\beta-(\beta-\alpha) w-\beta s)}\left(c^{2}|\kappa|^{2} t^{\beta}\right)^{-s} d s}_{\mathcal{I}}=H_{1,2}^{1,1}\left[\begin{array}{c|c}
c^{2}|\kappa|^{2} t^{\beta} & (s, 1) \\
(0,1),(-\beta+\beta s, \beta-\alpha)
\end{array}\right] .
$$


Moreover, since $\Delta=\beta-\alpha>0$ then the integral is convergent and the contour of integration $\mathcal{L}_{1}$ is transformed into the loop $\mathcal{L}_{-\infty}$ (cf. [12, Thm. 1.1]). As the gamma function $\Gamma(s)$ has simple poles at $s=-j$, for $j \in \mathbb{N}_{0}$, and $\operatorname{res}_{s=-j} \Gamma(s)=\frac{(-1)^{j}}{j !}$ then, by the Residue Theorem, we obtain that the integral $\mathcal{I}$ is equal to

$$
\mathcal{I}=\sum_{j=0}^{+\infty} \frac{(-1)^{j}}{j !}\left(c^{2}|\kappa|^{2} t^{\beta}\right)^{j} \frac{1}{2 \pi i} \int_{\mathcal{L}_{2}} \frac{\Gamma(1+j-w) \Gamma(w)}{\Gamma(1+\beta-(\beta-\alpha) w+\beta j)}\left(a t^{\beta-\alpha}\right)^{-w} d w
$$

Now we compute the integral with respect to $w$. Since

$$
\frac{1}{2 \pi i} \int_{\mathcal{L}_{2}} \frac{\Gamma(1+j-w) \Gamma(w)}{\Gamma(1+\beta-(\beta-\alpha) w+\beta j)}\left(a t^{\beta-\alpha}\right)^{-w} d w=H_{1,2}^{1,1}\left[a t^{\beta-\alpha} \mid \begin{array}{c}
(-j, 1) \\
(0,1),(-\beta-\beta j, \beta-\alpha)
\end{array}\right]
$$

and $\Delta=\beta-\alpha>0$ we conclude that the integral is convergent and $\mathcal{L}_{2}=\mathcal{L}_{-\infty}$ (cf. [12, Thm. 1.1]). Now, as the gamma function $\Gamma(w)$ has simple poles at $w=-k, k \in \mathbb{N}_{0}$ and $\operatorname{res}_{w=-k} \Gamma(w)=\frac{(-1)^{k}}{k !}$ then, applying the Residue Theorem to (21), we get the desire result

$$
\mathcal{I}=\sum_{j=0}^{+\infty} \sum_{k=0}^{+\infty} \frac{\Gamma(1+k+j)}{\Gamma(1+\beta+(\beta-\alpha) k+\beta j) k ! j !}\left(-a t^{\beta-\alpha}\right)^{k}\left(-c^{2}|\kappa|^{2} t^{\beta}\right)^{j} .
$$

From the previous lemma we get by immediate application of Definition 2.3 the following corollary.

Corollary 3.2 The multivariate Mittag-Leffler function in (17) has the following representation in the form of $H$-function of two variables

$$
E_{(\beta-\alpha, \beta), 1+\beta}\left(-a t^{\beta-\alpha},-c^{2}|\kappa|^{2} t^{\beta}\right)=H_{1,1 ; 0,1 ; 0,1}^{0,1 ; 1,0 ; 1,0}\left[\begin{array}{c|c}
a t^{\beta-\alpha} & (0 ; 1,1) ;-;- \\
c^{2}|\kappa|^{2} t^{\beta} & (-\beta ; \beta, \beta-\alpha) ;(0,1) ;(0,1)
\end{array}\right] .
$$

We observe that due to the conditions presented in [3] we can guarantee that the double Mellin-Barnes integral in (19) is convergent and the correspondent H-function of two variables (22) is analytic. Applying the inverse Fourier transform we get

$$
G_{n}^{\alpha, \beta}(x, t)=\frac{1}{(2 \pi)^{n}} \int_{\mathbb{R}^{n}} e^{-i \kappa \cdot x} \widehat{G}_{n}^{\alpha, \beta}(\kappa, t) d \kappa \quad x \in \mathbb{R}^{n}, t>0 .
$$

Since $\widehat{G}_{n}^{\alpha, \beta}(\kappa, t)$ is a radial function in $\kappa$ then the integral (23) can be reduced to radial integration. Using the following formula (see [18])

$$
\frac{1}{(2 \pi)^{n}} \int_{\mathbb{R}^{n}} e^{-i \kappa \cdot x} \phi(|\kappa|) d \kappa=\frac{|x|^{1-\frac{n}{2}}}{(2 \pi)^{\frac{n}{2}}} \int_{0}^{+\infty} \phi(\tau) \tau^{\frac{n}{2}} J_{\frac{n}{2}-1}(\tau|x|) d \tau,
$$

where $J_{\nu}$ represents the Bessel function of first kind with index $\nu$, we can write (23) as

$$
G_{n}^{\alpha, \beta}(x, t)=\frac{|x|^{1-\frac{n}{2}}}{(2 \pi)^{\frac{n}{2}}} \int_{0}^{+\infty} \tau^{\frac{n}{2}} \widehat{G}_{n}^{\alpha, \beta}(\tau, t) J_{\frac{n}{2}-1}(\tau|x|) d \tau .
$$

Taking into account (17) we get

$$
G_{n}^{\alpha, \beta}(x, t)=\delta(x)-\frac{|x|^{1-\frac{n}{2}} t^{\beta}}{(2 \pi)^{\frac{n}{2}}} \int_{0}^{+\infty} \tau^{\frac{n}{2}+2} E_{(\beta-\alpha, \beta), 1+\beta}\left(-a t^{\beta-\alpha},-c^{2} \tau^{2} t^{\beta}\right) J_{\frac{n}{2}-1}(\tau|x|) d \tau
$$

where $\delta(x)$ corresponds to the Dirac delta function in distributional sense. Taking into account (19), the second term in the right-hand side of $(26)$ is equal to

$$
-\frac{|x|^{1-\frac{n}{2}} t^{\beta}}{(2 \pi)^{\frac{n}{2}}(2 \pi i)^{2}} \int_{0}^{+\infty} \int_{\mathcal{L}_{1}} \int_{\mathcal{L}_{2}} \frac{\Gamma(-s) \Gamma(-w) \Gamma(1+s+w)}{\Gamma(1+(\beta-\alpha) w+\beta s)}\left(c^{2} \tau^{2} t^{\beta}\right)^{s}\left(a t^{\beta-\alpha}\right)^{w} J_{\frac{n}{2}-1}(\tau|x|) \tau^{\frac{n}{2}+2} d w d s
$$


Using Formula (7) in [7, p. 22] we have that

$$
\int_{0}^{+\infty} R^{\frac{n}{2}+2+2 s} J_{\frac{n}{2}-1}(R|x|) d R=\frac{2^{\frac{n}{2}+2+2 s}}{|x|^{\frac{n}{2}+3+2 s}} \frac{\Gamma\left(\frac{n}{2}+1+s\right)}{\Gamma(-1-s)},
$$

for $-\frac{n+2}{2}<\Re(s)<-\frac{n+4}{4}$. Therefore, (26) is equal to

$$
\begin{aligned}
G_{n}^{\alpha, \beta}(x, t)=\delta(x)- & \frac{4 c^{2} t^{\beta}}{|x|^{n+2} \pi^{\frac{n}{2}}} \frac{1}{(2 \pi i)^{2}} \int_{\mathcal{L}_{1}} \int_{\mathcal{L}_{2}} \frac{\Gamma(-s) \Gamma(-w) \Gamma(1+s+w) \Gamma\left(1+\frac{n}{2}+s\right)}{\Gamma(1+\beta+(\beta-\alpha) w+\beta s) \Gamma(-1-s)} \\
& \times\left(\frac{4 c^{2} t^{\beta}}{|x|^{2}}\right)^{s}\left(a t^{\beta-\alpha}\right)^{w} d w d s .
\end{aligned}
$$

Using the Definition 2.3, the double Mellin-Barnes type integral in (28) corresponds to the following H-function of two variables:

$$
H_{1,1 ; 0,1 ; 2,1}^{0,1 ; 1,0 ; 1,1}\left[\begin{array}{c|c}
a t^{\beta-\alpha} & (0 ; 1,1) ;-;\left(-\frac{n}{2}, 1\right),(-1,1) \\
4 c^{2} t^{\beta}|x|^{-2} & (-\beta ; \beta, \beta-\alpha) ;(0,1) ;(0,1)
\end{array}\right]
$$

Considering now the change of variables $s \mapsto-s$ and $w \mapsto-w$ and applying the general theory of the Fox H-function presented in [12] we realize that

$$
\frac{1}{2 \pi i} \int_{\mathcal{L}_{2}} \frac{\Gamma(1-s-w) \Gamma(w)}{\Gamma(1+\beta-(\beta-\alpha) w-\beta s)}\left(a t^{\beta-\alpha}\right)^{-w} d w=H_{1,1}^{1,2}\left[a t^{\beta-\alpha} \mid \begin{array}{c}
(s, 1) \\
(0,1),(-\beta-\beta s, \beta-\alpha)
\end{array}\right]
$$

is convergent and $\mathcal{L}_{2}=\mathcal{L}_{-\infty}$ since $\Delta=\beta-\alpha>0$ (cf. [12, Thm. 1.1]). As the gamma function $\Gamma(w)$ has simple poles at $w=-k, k \in \mathbb{N}_{0}$ and $\operatorname{res}_{w=-k} \Gamma(w)=\frac{(-1)^{k}}{k !}$ then, by the Residue Theorem, we get

$$
G_{n}^{\alpha, \beta}(x, t)=\delta(x)-\frac{4 c^{2} t^{\beta}}{\pi^{\frac{n}{2}}|x|^{n+2}} \sum_{k=0}^{+\infty} \frac{\left(-a t^{\beta-\alpha}\right)^{k}}{k !} \frac{1}{2 \pi i} \int_{\mathcal{L}_{1}} \frac{\Gamma(s) \Gamma(1-s+k) \Gamma\left(1+\frac{n}{2}-s\right)}{\Gamma(1+\beta+(\beta-\alpha) k-\beta s) \Gamma(s-1)}\left(\frac{4 c^{2} t^{\beta}}{|x|^{2}}\right)^{-s} d s
$$

To compute now the Mellin integral in order to $s$ we observe that

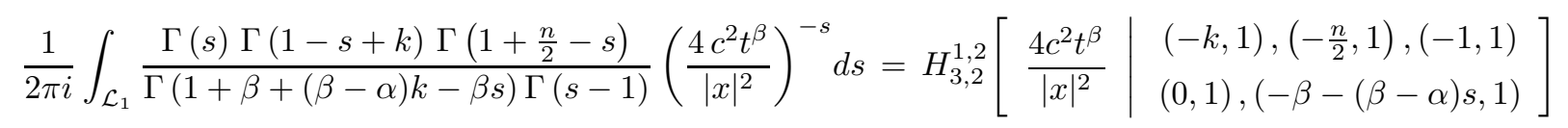

and since $\Delta=-1<0$ then contour of integration $\mathcal{L}_{1}$ must be transformed into the loop $\mathcal{L}_{+\infty}(\mathrm{cf}$. [12, Thm. 1.1]) starting and ending at infinity and encircling all poles of the functions $\Gamma(1-s+k)$ and $\Gamma\left(1+\frac{n}{2}-s\right)$.

Remark 3.3 To avoid the appearance of the delta Dirac function in (31) we could have used the following alternative representation for (17):

$$
\widehat{G}_{n}^{\alpha, \beta}(\kappa, t)=E_{(\beta-\alpha, \beta), 1}\left(-a t^{\beta-\alpha},-c^{2}|\kappa|^{2} t^{\beta}\right)+a t^{\beta-\alpha} E_{(\beta-\alpha, \beta), 1+\beta-\alpha}\left(-a t^{\beta-\alpha},-c^{2}|\kappa|^{2} t^{\beta}\right) .
$$

To see that (33) is equal to (17) we can simply expand and compare the terms of the series in both expressions. Applying the techniques presented in this section to (33) we get another representation of (31) given by:

$$
\begin{aligned}
G_{n}^{\alpha, \beta}(x, t)= & \frac{1}{\pi^{\frac{n}{2}}|x|^{n}} \sum_{k=0}^{+\infty} \frac{\left(-a t^{\beta-\alpha}\right)^{k}}{k !}\left[\frac{1}{2 \pi i} \int_{\mathcal{L}_{1}} \frac{\Gamma(1-s+k) \Gamma\left(\frac{n}{2}-s\right)}{\Gamma(1+(\beta-\alpha) k-\beta s)}\left(\frac{4 c^{2} t^{\beta}}{|x|^{2}}\right)^{-s} d s\right. \\
& \left.+\frac{a t^{\beta-\alpha}}{2 \pi i} \int_{\mathcal{L}_{1}} \frac{\Gamma(1-s+k) \Gamma\left(\frac{n}{2}-s\right)}{\Gamma(1+\beta-\alpha+(\beta-\alpha) k-\beta s)}\left(\frac{4 c^{2} t^{\beta}}{|x|^{2}}\right)^{-s} d s\right] .
\end{aligned}
$$

Since (31) will lead in the end to less double series we choose to work with it instead of the representation (34). Finally, considering $a=0$ in (33) and (34), we obtain immediately the correspondent expressions for the time-fractional wave operator deduced in [8].

To find a series representation for (32) we need to take into account the parity of the dimension $n$, as we will see in the next subsections. 


\subsection{The case of odd dimension}

From the analysis of (31) we conclude that for $n$ odd we have two non-coincident infinite sequences of simple poles at $s=j+k+1$, for $j \in \mathbb{N}_{0}$, and at $s=p+\frac{n}{2}+1$, for $p \in \mathbb{N}_{0}$, coming from the gamma functions $\Gamma(1-s+k)$ and $\Gamma\left(1+\frac{n}{2}-s\right)$, respectively. Therefore, applying the Residue Theorem we obtain the following series representation for the FS

$$
\begin{aligned}
G_{n}^{\alpha, \beta}(x, t)= & \delta(x)-\frac{4 c^{2} t^{\beta}}{\pi^{\frac{n}{2}}|x|^{n+2}} \sum_{k=0}^{+\infty} \frac{\left(-a t^{\beta-\alpha}\right)^{k}}{k !}\left[\sum_{j=0}^{+\infty} \frac{(-1)^{j}}{j !} \frac{\Gamma\left(\frac{n}{2}-j-k\right) \Gamma(j+k+1)}{\Gamma(1-\alpha k-\beta j) \Gamma(j+k)}\left(\frac{4 c^{2} t^{\beta}}{|x|^{2}}\right)^{-j-k-1}\right. \\
& \left.+\sum_{p=0}^{+\infty} \frac{(-1)^{p}}{p !} \frac{\Gamma\left(k-p-\frac{n}{2}\right) \Gamma\left(p+\frac{n}{2}+1\right)}{\Gamma\left(1+(\beta-\alpha) k-\beta\left(p+\frac{n}{2}\right)\right) \Gamma\left(p+\frac{n}{2}\right)}\left(\frac{4 c^{2} t^{\beta}}{|x|^{2}}\right)^{-p-\frac{n}{2}-1}\right]
\end{aligned}
$$

By (3) we have that

$$
\frac{\Gamma(j+k+1)}{\Gamma(j+k)}=j+k \quad \text { and } \quad \frac{\Gamma\left(p+\frac{n}{2}+1\right)}{\Gamma\left(p+\frac{n}{2}\right)}=p+\frac{n}{2} .
$$

Therefore, (35) simplifies to

$$
\begin{aligned}
G_{n}^{\alpha, \beta}(x, t)= & \delta(x)-\frac{1}{\pi^{\frac{n}{2}}|x|^{n}} \sum_{k=0}^{+\infty} \frac{\left(-a t^{\beta-\alpha}\right)^{k}}{k !}\left[\sum_{j=0}^{+\infty} \frac{(-1)^{j}}{j !} \frac{\Gamma\left(\frac{n}{2}-j-k\right)(j+k)}{\Gamma(1-\alpha k-\beta j)}\left(\frac{|x|^{2}}{4 c^{2} t^{\beta}}\right)^{j+k}\right. \\
& \left.+\sum_{p=0}^{+\infty} \frac{(-1)^{p}}{p !} \frac{\Gamma\left(k-p-\frac{n}{2}\right)\left(p+\frac{n}{2}\right)}{\Gamma\left(1+(\beta-\alpha) k-\beta\left(p+\frac{n}{2}\right)\right)}\left(\frac{|x|^{2}}{4 c^{2} t^{\beta}}\right)^{p+\frac{n}{2}}\right]
\end{aligned}
$$

The series in brackets can be combined in one single series. For obtaining this we start by considering the change of variables $m=2 j+1$ and $m=2 p$ in the first and the second series in brackets, respectively. Hence, we get

$$
\begin{aligned}
G_{n}^{\alpha, \beta}(x, t)=\delta(x) & -\frac{1}{\pi^{\frac{n}{2}}|x|^{n}} \sum_{k=0}^{+\infty} \frac{\left(-a t^{\beta-\alpha}\right)^{k}}{k !}\left[\sum_{\substack{m=1 \\
m \text { odd }}}^{+\infty} \frac{(-1)^{\frac{m-1}{2}}}{\Gamma\left(\frac{m-1}{2}+1\right)} \frac{\Gamma\left(\frac{n}{2}-\frac{m-1}{2}-k\right)\left(\frac{m-1}{2}+k\right)}{\Gamma\left(1-\alpha k-\frac{\beta(m-1)}{2}\right)}\left(\frac{|x|^{2}}{4 c^{2} t^{\beta}}\right)^{\frac{m-1}{2}+k}\right. \\
& \left.+\sum_{\substack{m=0 \\
m \text { even }}}^{+\infty} \frac{(-1)^{\frac{m}{2}}}{\Gamma\left(\frac{m}{2}+1\right)} \frac{\Gamma\left(k-\frac{m}{2}-\frac{n}{2}\right)\left(\frac{m+n}{2}\right)}{\Gamma\left(1+(\beta-\alpha) k-\frac{\beta(m+n)}{2}\right)}\left(\frac{|x|^{2}}{4 c^{2} t^{\beta}}\right)^{\frac{m+n}{2}}\right] .
\end{aligned}
$$

To have the same exponent in both series we consider the changes $m=p+n+1-2 k$ and $m=p$ in the first and the second series in brackets, yielding

$$
\begin{aligned}
G_{n}^{\alpha, \beta}(x, t)= & \delta(x)-\frac{1}{\pi^{\frac{n}{2}}|x|^{n}} \sum_{k=0}^{+\infty} \frac{\left(-a t^{\beta-\alpha}\right)^{k}}{k !}\left[\sum_{\substack{p=2 k-n \\
\text { podd }}}^{-1} \frac{(-1)^{\frac{p+n}{2}-k}}{\Gamma\left(\frac{p+n}{2}-k+1\right)} \frac{\Gamma\left(-\frac{p}{2}\right)\left(\frac{p+n}{2}\right)}{\Gamma\left(1+(\beta-\alpha) k-\frac{\beta(p+n)}{2}\right)}\left(\frac{|x|^{2}}{4 c^{2} t^{\beta}}\right)^{\frac{p+n}{2}}\right. \\
& +\sum_{\substack{p=1 \\
p \text { odd }}}^{+\infty} \frac{(-1)^{\frac{p+n}{2}}-k}{\Gamma\left(\frac{p+n}{2}-k+1\right)} \frac{\Gamma\left(-\frac{p}{2}\right)\left(\frac{p+n}{2}\right)}{\Gamma\left(1+(\beta-\alpha) k-\frac{\beta(p+n)}{2}\right)}\left(\frac{|x|^{2}}{4 c^{2} t^{\beta}}\right)^{\frac{p+n}{2}} \\
& \left.+\sum_{\substack{p=0 \\
p \text { even }}}^{+\infty} \frac{(-1)^{\frac{p}{2}}}{\Gamma\left(\frac{p}{2}+1\right)} \frac{\Gamma\left(k-\frac{n+p}{2}\right)\left(\frac{p+n}{2}\right)}{\Gamma\left(1+(\beta-\alpha) k-\frac{\beta(p+n)}{2}\right)}\left(\frac{|x|^{2}}{4 c^{2} t^{\beta}}\right)^{\frac{p+n}{2}}\right]
\end{aligned}
$$

Now, we analyse the coefficients of the odd and the even series. For $p$ odd, using (4) and after straightforward calculations we obtain

$$
\frac{(-1)^{\frac{p+n}{2}-k} \Gamma\left(-\frac{p}{2}\right)}{\Gamma\left(\frac{p+n}{2}-k+1\right)}=\frac{(-1)^{\frac{p+n-2}{2}} \Gamma\left(-\frac{p}{2}\right)\left(1-\frac{p+n}{2}\right)_{k-1}}{\Gamma\left(\frac{p+n}{2}\right)} .
$$


Considering now the following relation proved in [8, Sec. 3.2.1] for $p$ odd

$$
\frac{(-1)^{\frac{p+n-2}{2}} \Gamma\left(-\frac{p}{2}\right)}{\Gamma\left(\frac{p+n}{2}\right)}=-\frac{(-1)^{\frac{n-1}{2}} \sqrt{\pi} 2^{p}}{\left(\frac{p+1}{2}\right)_{\frac{n-1}{2}} p !}
$$

we conclude that

$$
\frac{(-1)^{\frac{p+n}{2}-k} \Gamma\left(-\frac{p}{2}\right)}{\Gamma\left(\frac{p+n}{2}-k+1\right)}=-\frac{(-1)^{\frac{n-1}{2}} \sqrt{\pi} 2^{p}\left(1-\frac{p+n}{2}\right)_{k-1}}{p !\left(\frac{p+1}{2}\right)_{\frac{n-1}{2}}} .
$$

On the other hand, for $p$ even, using (3) and after straightforward calculations we obtain

$$
\frac{(-1)^{\frac{p}{2}} \Gamma\left(k-\frac{p+n}{2}\right)}{\Gamma\left(\frac{p}{2}+1\right)}=\frac{(-1)^{\frac{p}{2}} \Gamma\left(1-\frac{p+n}{2}\right)\left(1-\frac{p+n}{2}\right)_{k-1}}{\Gamma\left(\frac{p}{2}+1\right)} .
$$

Considering now the following relation proved in [8, Sec. 3.2.1] for $p$ even

$$
\frac{(-1)^{\frac{p}{2}} \Gamma\left(1-\frac{n}{2}-\frac{p}{2}\right)}{\Gamma\left(\frac{p}{2}+1\right)}=\frac{(-1)^{\frac{n-1}{2}} \sqrt{\pi} 2^{p}}{\left(\frac{p+1}{2}\right)_{\frac{n-1}{2}} p !},
$$

we conclude that

$$
\frac{(-1)^{\frac{p}{2}} \Gamma\left(k-\frac{p+n}{2}\right)}{\Gamma\left(\frac{p}{2}+1\right)}=\frac{(-1)^{\frac{n-1}{2}} \sqrt{\pi} 2^{p}\left(1-\frac{p+n}{2}\right)_{k-1}}{p !\left(\frac{p+1}{2}\right)_{\frac{n-1}{2}}} .
$$

From (37) and (39) we see that the coefficients of the series in brackets are equal up to a minus sign in the odd series, which can be included as $(-1)^{p}$ for $p$ odd and even. Therefore, summing the even and odd series and considering the change $p=2 q-n+2 k$ in the finit sum, we get the simplified series representation for the FS

$$
\begin{aligned}
G_{n}^{\alpha, \beta}(x, t)= & \delta(x)-\frac{1}{\pi^{\frac{n}{2}}|x|^{n}} \sum_{k=0}^{+\infty} \frac{\left(-a t^{\beta-\alpha}\right)^{k}}{k !}\left[\sum_{q=0}^{\frac{n-2 k-1}{2}} \frac{(-1)^{q}}{q !} \frac{\Gamma\left(\frac{n}{2}-q-k\right)(q+k)}{\Gamma(1-\beta q-\alpha k)}\left(\frac{|x|^{2}}{4 c^{2} t^{\beta}}\right)^{q+k}\right. \\
& \left.+\frac{(-1)^{\frac{n-1}{2}} \sqrt{\pi}|x|^{n}}{\left(4 c^{2} t^{\beta}\right)^{\frac{n}{2}}} \sum_{p=0}^{+\infty} \frac{\left(\frac{p+n}{2}\right)\left(1-\frac{p+n}{2}\right)_{k-1}}{\left(\frac{p+1}{2}\right)_{\frac{n-1}{2}} \Gamma\left(1+(\beta-\alpha) k-\frac{\beta(p+n)}{2}\right) p !}\left(-\frac{|x|}{c t^{\frac{\beta}{2}}}\right)^{p}\right] .
\end{aligned}
$$

From (13) we conclude that in the odd case the function $G_{n}^{\alpha, \beta}$ has the following asymptotic behaviour at infinity

$$
G_{n}^{\alpha, \beta}(x, t)=O\left(t^{-\frac{\beta n}{2}}\right) .
$$

Remark 3.4 If we consider $a=0$ in (40), we get (up to the singular term) the FS of the time-fractional wave operator deduced in [8, Sec. 3.2.1] for the case of $n$ odd, which is

$$
\begin{aligned}
G_{n}^{\beta}(x, t)= & \frac{1}{4 c^{2} \pi^{\frac{n}{2}}|x|^{n-2} t^{\beta}} \sum_{q=0}^{\frac{n-3}{2}} \frac{\Gamma\left(-1-q+\frac{n}{2}\right)}{\Gamma(1-\beta(q+1)) q !}\left(-\frac{|x|^{2}}{4 c^{2} t^{\beta}}\right)^{q} \\
& +\frac{(-1)^{\frac{n-1}{2}} \sqrt{\pi}}{\left(4 \pi c^{2} t^{\beta}\right)^{\frac{n}{2}}} \sum_{p=0}^{+\infty} \frac{1}{\left(\frac{p+1}{2}\right)_{\frac{n-1}{2}} \Gamma\left(1-\frac{\beta(p+n)}{2}\right) p !}\left(-\frac{|x|}{c t^{\frac{\beta}{2}}}\right)^{p} .
\end{aligned}
$$

\subsection{The case of even dimension}

For the case of even dimension we consider first the change of variables $1-s+k=u$ in the integral (31) yielding

$$
G_{n}^{\alpha, \beta}(x, t)=\delta(x)-\frac{4 c^{2} t^{\beta}}{\pi^{\frac{n}{2}}|x|^{n+2}} \sum_{k=0}^{+\infty} \frac{\left(-a t^{\beta-\alpha}\right)^{k}}{k !} \frac{1}{2 \pi i} \int_{\mathcal{L}_{1}^{*}} \frac{\Gamma(1+k-u) \Gamma(u) \Gamma\left(u-k+\frac{n}{2}\right)}{\Gamma(1-\alpha k+\beta u) \Gamma(k-u)}\left(\frac{4 c^{2} t^{\beta}}{|x|^{2}}\right)^{u-k-1} d u
$$

We have to consider now the infinite loop $\mathcal{L}_{1}^{*}=\mathcal{L}_{-\infty}$. Analysing the poles of the gamma functions $\Gamma(u)$ and $\Gamma\left(u-k+\frac{n}{2}\right)$ we conclude that the function $\Gamma(u)$ has poles at $u=-i$, for $i \in \mathbb{N}_{0}$ and the function $\Gamma\left(u-k+\frac{n}{2}\right)$ has poles at $u=k-\frac{n}{2}-j$, for $j \in \mathbb{N}_{0}$. Since $n$ is even we can have simple and/or double poles in the following three cases: 
- for $k-\frac{n}{2}<0$ there are simple poles at $u=-i$, for $i=0, \ldots,-k+\frac{n}{2}-1$ and double poles at $u=-i$, for $i \geq-k+\frac{n}{2}$

- for $k-\frac{n}{2}=0$ there are only double poles at $u=-i$, for $i \in \mathbb{N}_{0}$;

- for $k-\frac{n}{2}>0$ there are simple poles at $u=k-\frac{n}{2}-j$, for $j=0, \ldots, k-\frac{n}{2}-1$ and double poles at $u=k-\frac{n}{2}-j$, for $j \geq k-\frac{n}{2}$.

Therefore, applying the Residue Theorem and splitting the series in $k$ we obtain the following series representation for the FS:

$$
\begin{aligned}
& G_{n}^{\alpha, \beta}(x, t)=\delta(x)-\frac{4 c^{2} t^{\beta}}{\pi^{\frac{n}{2}}|x|^{n+2}}\left\{\sum _ { k = 0 } ^ { \frac { n } { 2 } - 1 } \frac { ( - a t ^ { \beta - \alpha } ) ^ { k } } { k ! } \left[\sum_{i=0}^{\frac{n}{2}-k-1} \frac{(-1)^{i}}{i !} \frac{\Gamma\left(-i-k+\frac{n}{2}\right)(i+k)}{\Gamma(1-\alpha k-\beta i)}\left(\frac{|x|^{2}}{4 c^{2} t^{\beta}}\right)^{i+k+1}\right.\right. \\
& \left.+\sum_{i=\frac{n}{2}-k}^{+\infty} \frac{(-1)^{k+\frac{n}{2}} A_{1}}{i !\left(i+k-\frac{n}{2}\right) ! \Gamma(1-\alpha k-\beta i)}\left(\frac{|x|^{2}}{4 c^{2} t^{\beta}}\right)^{i+k+1}\right]+\frac{\left(-a t^{\beta-\alpha}\right)^{\frac{n}{2}}}{\left(\frac{n}{2}\right) !} \sum_{j=0}^{+\infty} \frac{A_{2}}{(j !)^{2} \Gamma\left(1-\beta j-\frac{\alpha n}{2}\right)}\left(\frac{|x|^{2}}{4 c^{2} t^{\beta}}\right)^{j+\frac{n}{2}+1} \\
& +\sum_{k=\frac{n}{2}+1}^{+\infty} \frac{\left(-a t^{\beta-\alpha}\right)^{k}}{k !}\left[\sum_{j=0}^{k-\frac{n}{2}-1} \frac{(-1)^{j}}{j !} \frac{\Gamma\left(-j+k-\frac{n}{2}\right)\left(j+\frac{n}{2}\right)}{\Gamma\left(1-\beta\left(j-k+\frac{n}{2}\right)-\alpha k\right)}\left(\frac{|x|^{2}}{4 c^{2} t^{\beta}}\right)^{j+\frac{n}{2}+1}\right. \\
& \left.\left.+\sum_{j=k-\frac{n}{2}}^{+\infty} \frac{(-1)^{k+\frac{n}{2}} A_{3}}{j !\left(j-k+\frac{n}{2}\right) ! \Gamma\left(1-\beta\left(j-k+\frac{n}{2}\right)-\alpha k\right)}\left(\frac{|x|^{2}}{4 c^{2} t^{\beta}}\right)^{j+\frac{n}{2}+1}\right]\right\}
\end{aligned}
$$

where $A_{1}, A_{2}$ and $A_{3}$ are the following expressions

$$
\begin{aligned}
& A_{1}=-1+(i+k)\left(\ln \left(\frac{4 c^{2} t^{\beta}}{|x|^{2}}\right)+\psi(1+i)+\psi\left(1+i+k-\frac{n}{2}\right)-\beta \psi(1-\alpha k-\beta i)\right), \\
& A_{2}=-1+\left(j+\frac{n}{2}\right)\left(\ln \left(\frac{4 c^{2} t^{\beta}}{|x|^{2}}\right)+2 \psi(1+j)-\beta \psi\left(1-\beta j-\frac{\alpha n}{2}\right)\right), \\
& A_{3}=-1+\left(j+\frac{n}{2}\right)\left(\ln \left(\frac{4 c^{2} t^{\beta}}{|x|^{2}}\right)+\psi(1+j)+\psi\left(1+j-k+\frac{n}{2}\right)-\beta \psi\left(1-\beta\left(j-k+\frac{n}{2}\right)-\alpha k\right)\right),
\end{aligned}
$$

and $\psi(z)$ denotes the digamma function. From the previous expression we see that the series of residues obtained for the case $k=\frac{n}{2}$ can be included as a limit case in the series of residues obtained for the case $k>\frac{n}{2}$. Moreover, rearranging the inner series we obtain the simplified expression

$$
\begin{aligned}
G_{n}^{\alpha, \beta}(x, t)= & \delta(x)-\frac{4 c^{2} t^{\beta}}{\pi^{\frac{n}{2}}|x|^{n+2}}\left\{\sum _ { k = 0 } ^ { \frac { n } { 2 } - 1 } \frac { ( - a t ^ { \beta - \alpha } ) ^ { k } } { k ! } \left[\sum_{i=0}^{\frac{n}{2}-k-1} \frac{(-1)^{i}}{i !} \frac{\Gamma\left(-i-k+\frac{n}{2}\right)(i+k)}{\Gamma(1-\beta i-\alpha k)}\left(\frac{|x|^{2}}{4 c^{2} t^{\beta}}\right)^{i+k+1}\right.\right. \\
& \left.+\sum_{i=0}^{+\infty} \frac{(-1)^{k+\frac{n}{2}} A_{4}}{i !\left(i-k+\frac{n}{2}\right) ! \Gamma\left(1-\beta\left(i-k+\frac{n}{2}\right)-\alpha k\right)}\left(\frac{|x|^{2}}{4 c^{2} t^{\beta}}\right)^{i+\frac{n}{2}+1}\right] \\
& +\sum_{k=\frac{n}{2}}^{+\infty} \frac{\left(-a t^{\beta-\alpha}\right)^{k}}{k !}\left[\sum_{j=0}^{k-\frac{n}{2}-1} \frac{(-1)^{j}}{j !} \frac{\Gamma\left(-j+k-\frac{n}{2}\right)\left(j+\frac{n}{2}\right)}{\Gamma\left(1-\beta\left(j-k+\frac{n}{2}\right)-\alpha k\right)}\left(\frac{|x|^{2}}{4 c^{2} t^{\beta}}\right)^{j+\frac{n}{2}+1}\right. \\
& \left.\left.+\sum_{j=0}^{+\infty} \frac{(-1)^{k+\frac{n}{2}} A_{5}}{j !\left(j+k-\frac{n}{2}\right) ! \Gamma(1-\beta j-\alpha k)}\left(\frac{|x|^{2}}{4 c^{2} t^{\beta}}\right)^{j+k+1}\right]\right\}
\end{aligned}
$$

where $A_{4}$ and $A_{5}$ are the following expressions

$$
\begin{aligned}
& A_{4}=-1+\left(i+\frac{n}{2}\right)\left(\ln \left(\frac{4 c^{2} t^{\beta}}{|x|^{2}}\right)+\psi\left(1+i-k+\frac{n}{2}\right)+\psi(1+i)-\beta \psi\left(1-\beta\left(i-k+\frac{n}{2}\right)-\alpha k\right)\right), \\
& A_{5}=-1+(j+k)\left(\ln \left(\frac{4 c^{2} t^{\beta}}{|x|^{2}}\right)+\psi\left(1+j+k-\frac{n}{2}\right)+\psi(1+j)-\beta \psi(1-\beta j-\alpha k)\right) .
\end{aligned}
$$

Remark 3.5 For some rational values of $\beta$ we have an indetermination in the series coefficients due to the terms $\beta \psi\left(1-\beta\left(i-k+\frac{n}{2}\right)-\alpha k\right)$ and $\Gamma\left(1-\beta\left(i-k+\frac{n}{2}\right)-\alpha k\right)$ in the first inner series, and due to the terms 
$\beta \psi(1-\beta j-\alpha k)$ and $\Gamma(1-\beta j-\alpha k)$ in the second inner series. These indeterminations can be removed after applying (3) with $n=1,(6)$, (7), and the relation $\psi(1-z)=\pi \cot (\pi z)+\psi(z)$ for the digamma function. Moreover, if we consider $a=0$ in (43) we get (up to the singular term) the FS of the time-fractional wave operator deduced in [8, Sec. 3.2.2] for the case of $n$ even, which is

$$
\begin{aligned}
G_{n}^{\beta}(x, t)= & \frac{1}{4 c^{2} \pi^{\frac{n}{2}}|x|^{n-2} t^{\beta}} \sum_{i=0}^{\frac{n}{2}-2} \frac{\Gamma\left(\frac{n}{2}-i-1\right)}{\Gamma(1-\beta(i+1)) i !}\left(-\frac{|x|^{2}}{4 c^{2} t^{\beta}}\right)^{i} \\
& +\frac{(-1)^{\frac{n}{2}+1}}{\left(4 \pi c^{2} t^{\beta}\right)^{\frac{n}{2}}} \sum_{i=0}^{+\infty} \frac{\psi\left(i+\frac{n}{2}\right)-\beta \psi\left(1-\beta\left(i+\frac{n}{2}\right)\right)+\psi(i+1)+\ln \left(\frac{4 c^{2} t^{\beta}}{|x|^{2}}\right)}{\Gamma\left(i+\frac{n}{2}\right) \Gamma\left(1-\beta\left(i+\frac{n}{2}\right)\right) i !}\left(\frac{|x|^{2}}{4 c^{2} t^{\beta}}\right)^{i} .
\end{aligned}
$$

\section{Moments}

In this section we obtain the expression for some moments of the FS $G_{n}^{\alpha, \beta}$. Our approach consists in a reinterpretation of the moments in terms of the Laplace transform (see e.g. [16]). Applying the Laplace transform with respect to the variable $t$ to (16) and taking into account the initial conditions and Lemma 2.24 in [11], we get

$$
\mathcal{L}\left\{\widehat{G}_{n}^{\alpha, \beta}\right\}(r, s)=\frac{s^{\beta-1}+a s^{\alpha-1}}{s^{\beta}+a s^{\alpha}+c^{2} r^{2}},
$$

where $r=|\kappa|, 0<\alpha \leq 1,1<\beta \leq 2$. Now, computing the integer derivative of order $\gamma>0$ of (44) with respect to $r$ at $r=0$, we get

$$
D_{r}^{\gamma}\left[\mathcal{L}\left\{\widehat{G}_{n}^{\alpha, \beta}\right\}\right](0, s)=\left\{\begin{array}{cl}
0, & \gamma \text { is odd } \\
\frac{(-1)^{\frac{\gamma}{2}} \gamma ! c^{\gamma}}{s\left(a s^{\alpha}+s^{\beta}\right)^{\frac{\gamma}{2}}}, & \gamma \text { is even }
\end{array} .\right.
$$

On the other hand, taking into account the definition of the Fourier transform for radial functions in $\mathbb{R}^{n}$ (see [18]) and due to the convergence of the improper integrals, we have

$$
\begin{aligned}
& D_{r}^{\gamma}\left[\mathcal{L}\left\{\widehat{G}_{n}^{\alpha, \beta}\right\}\right](0, s)=D_{r}^{\gamma}[\int_{0}^{+\infty} e^{-s t} \underbrace{\frac{(2 \pi)^{\frac{n}{2}}}{r^{\frac{n}{2}-1}} \int_{0}^{+\infty} G_{n}^{\alpha, \beta}(w, t) J_{\frac{n}{2}-1}(w r) w^{\frac{n}{2}} d w}_{\widehat{G}_{n}^{\alpha, \beta}(r, t)} d t](0, s) \\
& =\int_{0}^{+\infty} e^{-s t} \int_{0}^{+\infty} D_{r}^{\gamma}\left[\frac{(2 \pi)^{\frac{n}{2}}}{r^{\frac{n}{2}-1}} J_{\frac{n}{2}-1}(w r) w^{\frac{n}{2}}\right]_{r=0} G_{n}^{\alpha, \beta}(w, t) d w d t .
\end{aligned}
$$

Since

$$
D_{r}^{\gamma}\left[\frac{(2 \pi)^{\frac{n}{2}}}{r^{\frac{n}{2}-1}} J_{\frac{n}{2}-1}(w r) w^{\frac{n}{2}}\right]_{r=0}=\left\{\begin{array}{cl}
0, & \gamma \text { is odd } \\
\frac{(-1)^{\frac{\gamma}{2}}(\gamma-1) ! ! \pi^{\frac{n}{2}} w^{n+\gamma-1}}{2^{\frac{\gamma}{2}-1} \Gamma\left(\frac{\gamma+n}{2}\right)}, & \gamma \text { is even }
\end{array},\right.
$$

then when $\gamma$ is even (46) becomes

$$
\begin{aligned}
D_{r}^{\gamma}\left[\mathcal{L}\left\{\widehat{G}_{n}^{\alpha, \beta}\right\}\right](0, s) & =\frac{(-1)^{\frac{\gamma}{2}}(\gamma-1) ! ! \pi^{\frac{n}{2}}}{2^{\frac{\gamma}{2}-1} \Gamma\left(\frac{\gamma+n}{2}\right)} \int_{0}^{+\infty} e^{-s t} \int_{0}^{+\infty} w^{n+\gamma-1} G_{n}^{\alpha, \beta}(w, t) d w d t \\
& =\frac{(-1)^{\frac{\gamma}{2}}(\gamma-1) ! ! \pi^{\frac{n}{2}}}{2^{\frac{\gamma}{2}-1} \Gamma\left(\frac{\gamma+n}{2}\right)} \mathcal{L}\left\{\mathbf{M}_{\alpha, \beta, n}^{n+\gamma-1}\right\}(s),
\end{aligned}
$$

where $\mathbf{M}_{\alpha, \beta, n}^{n+\gamma-1}$ is the moment of order $n+\gamma+1$ of $G_{n}^{\alpha, \beta}$. Moreover, from (45) and (47) we get

$$
\mathcal{L}\left\{\mathbf{M}_{\alpha, \beta, n}^{n+\gamma-1}\right\}(s)=\frac{\gamma ! ! 2^{\frac{\gamma}{2}-1} c^{\gamma} \Gamma\left(\frac{\gamma+n}{2}\right)}{\pi^{\frac{n}{2}} s\left(a s^{\alpha}+s^{\beta}\right)^{\frac{\gamma}{2}}} .
$$

Inverting the Laplace transform, we have

$$
\mathbf{M}_{\alpha, \beta, n}^{n+\gamma-1}(t)=\frac{\gamma ! ! 2^{\frac{\gamma}{2}-1} c^{\gamma} \Gamma\left(\frac{\gamma+n}{2}\right)}{\pi^{\frac{n}{2}}} \mathcal{L}^{-1}\left\{\frac{1}{s\left(a s^{\alpha}+s^{\beta}\right)^{\frac{\gamma}{2}}}\right\}(t) .
$$


Taking into account formula (5.1.26) presented in [9]

$$
\mathcal{L}\left\{t^{\nu} E_{\mu, \nu}^{\rho}\left(\lambda t^{\mu}\right)\right\}(s)=\frac{s^{\rho \mu-\nu}}{\left(s^{\mu}-\lambda\right)^{\rho}}, \quad \Re(s)>0, \quad \Re(\nu)>0, \quad \lambda \in \mathbb{C}, \quad\left|\lambda s^{-\mu}\right|<1,
$$

where $E_{\mu, \nu}^{\rho}$ is the three-parameter Mittag-Leffler function defined by the series (see [9])

$$
E_{\mu, \nu}^{\rho}(z)=\sum_{k=0}^{+\infty} \frac{(\rho)_{k}}{\Gamma(\mu k+\nu) k !} z^{k}
$$

we finally conclude that the moments of order $n+\gamma+1$ of $G_{n}^{\alpha, \beta}$ are given by

$$
\mathbf{M}_{\alpha, \beta, n}^{n+\gamma-1}(t)=\frac{\gamma ! ! 2^{\frac{\gamma}{2}-1} c^{\gamma} \Gamma\left(\frac{\gamma+n}{2}\right)}{\pi^{\frac{n}{2}}} t^{\frac{\beta \gamma}{2}} E_{\beta-\alpha, \frac{\beta \gamma}{2}+1}^{\frac{\gamma}{2}}\left(-a t^{\beta-\alpha}\right)
$$

where $\gamma$ is a non-negative even integer. We observe that with our approach it is not possible to compute the moment of order 0 and, therefore, we cannot guarantee that $G_{n}^{\alpha, \beta}$ is a probability density function. In expression (49) if $\mu=m \in \mathbb{N}$ the function $E_{m, \nu}^{\rho}$ takes the following particular form (see formula (5.1.39) in [9])

$$
E_{m, \nu}^{\rho}(z)=\frac{1}{\Gamma(\nu)}{ }_{1} F_{m}\left(\rho ; \frac{\nu}{m}, \frac{\nu+1}{m}, \ldots, \frac{\nu+m-1}{m} ; \frac{z}{m^{m}}\right),
$$

where ${ }_{1} F_{m}$ is the generalized hypergeometric function. In our case, due to the fact that $0<\alpha \leq 1$ and $1<\beta \leq 2$ it is possible to have $\beta-\alpha=1$ for some values of $\alpha$ and $\beta$. In these cases we can apply the previous formula, obtaining

$$
\begin{aligned}
\mathbf{M}_{\alpha, 1+\alpha, n}^{n+\gamma-1}(t) & =\frac{\gamma ! ! 2^{\frac{\gamma}{2}-1} c^{\gamma} \Gamma\left(\frac{\gamma+n}{2}\right)}{\pi^{\frac{n}{2}} \Gamma\left(1+\frac{(1+\alpha) \gamma}{2}\right)} t^{\frac{(1+\alpha) \gamma}{2}}{ }_{1} F_{1}\left(\frac{\gamma}{2} ; 1+\frac{(1+\alpha) \gamma}{2} ;-a t\right) \\
& =\frac{\gamma ! ! 2^{\frac{\gamma}{2}-1} c^{\gamma} \Gamma\left(\frac{\gamma+n}{2}\right)}{\pi^{\frac{n}{2}} \Gamma\left(1+\frac{(1+\alpha) \gamma}{2}\right)} t^{\frac{(1+\alpha) \gamma}{2}} \Phi\left(\frac{\gamma}{2} ; 1+\frac{(1+\alpha) \gamma}{2} ;-a t\right)
\end{aligned}
$$

where $\Phi(\rho ; \nu ; z)$ is the Kummer confluent hypergeometric function.

Remark 4.1 If we consider $\gamma=2, \beta=2 \alpha$ and $n=1$ in (50) we obtain the expression deduced in [16] for the variance of the FS, i.e.,

$$
\mathbf{M}_{\alpha, 2 \alpha, 1}^{2}(t)=2 c^{2} t^{2 \alpha} E_{\alpha, 2 \alpha+1}\left(-a t^{\alpha}\right)
$$

Remark 4.2 Considering $a=0$ in (50) and taking into account (49), we get

$$
\mathbf{M}_{\alpha, \beta, n}^{n+\gamma-1}(t)=\frac{\gamma ! ! 2^{\frac{\gamma}{2}-1} c^{\gamma} \Gamma\left(\frac{\gamma+n}{2}\right)}{\pi^{\frac{n}{2}}} t^{\frac{\beta \gamma}{2}} \frac{1}{\Gamma\left(1+\frac{\beta \gamma}{2}\right)}
$$

Using the following relation for the double factorial (see [1])

$$
m ! !=2^{\frac{m}{2}}\left(\frac{\pi}{2}\right)^{\frac{1}{4}(\cos (m \pi)-1)} \Gamma\left(1+\frac{m}{2}\right), \quad m \in \mathbb{N},
$$

and after straightforward calculations, expression (51) becomes

$$
\mathbf{M}_{\alpha, \beta, n}^{n+\gamma-1}(t)=\frac{2^{\gamma-1} c^{\gamma} \Gamma\left(1+\frac{\gamma}{2}\right) \Gamma\left(\frac{n+\gamma}{2}\right)}{\pi^{\frac{n}{2}} \Gamma\left(1+\frac{\beta \gamma}{2}\right)} t^{\frac{\beta \gamma}{2}}
$$

which corresponds to the moment of order $n+\gamma+1$, with $\gamma$ a non negative even integer, of the FS of the time fractional diffusion-wave equation studied in [8].

We observe that this approach does not give all the moments for any dimension. For example, when $n=1$ it is only possible to calculate the moments of even order, while for $n=2$ we can only calculate the moments of odd order. 


\section{An application to the law of a telegraph process with Brownian time}

In this section we study a particular case of the time-fractional telegraph equation. Let us consider in the one dimensional case the following fractional differential equation with $\beta=2 \alpha, 0<\alpha \leq 1$, and $a=2 \lambda \geq 0$ given by:

$$
D_{t}^{2 \alpha} u(x, t)+2 \lambda D_{t}^{\alpha} u(x, t)=c^{2} \partial_{x x}^{2} u(x, t),
$$

where $x \in \mathbb{R}, t>0, c>0$, and subject, for $0<\alpha \leq \frac{1}{2}$ to the initial condition $u(x, 0)=\delta(x)$ while, for $\frac{1}{2}<\alpha \leq 1$, besides the previous condition, also $u_{t}(x, 0)=0$ is assumed. Equation (52) was already studied in [16], where the authors presented only an integral representation for the Fourier transform of the FS. Moreover, for $\alpha=\frac{1}{2}$, it was obtained an integral representation of the FS based on the Fourier inversion transform (see Theorem 4.2 in [16]). Here we present an explicit series representation for such FS. Since our previous results are still valid when $0<\alpha \leq 1$ and $\alpha<\beta \leq 2$, we can consider in (40) $\beta=2 \alpha$ with $0<\alpha \leq 1$ and $n=1$, which leads to the following explicit series representation of the FS of (52)

$$
G_{1}^{\alpha, 2 \alpha}(x, t)=\delta(x)+\frac{1}{2 c t^{\alpha}} \sum_{k=0}^{+\infty} \frac{\left(-2 \lambda t^{\alpha}\right)^{k}}{k !} \sum_{p=0}^{+\infty} \frac{\left(-\frac{p+1}{2}\right)_{k}}{\Gamma(1+\alpha(k-p-1))}\left(-\frac{|x|}{c t^{\alpha}}\right)^{p},
$$

It is known that this function can be interpreted as probability density function (see [16]). In Section 6 we present some plot of the FS for $n=1$.

\subsection{Particular case of $\alpha=\frac{1}{2}$}

If we consider $\alpha=\frac{1}{2}$ in (52), we obtain a heat equation with damping term which depends on all values of $u$ in $[0, t]$ and assigning an overwhelming weight to those close to $t$ (see [16]). The damping effect of the fractional derivative reverberates on the distribution $u$, where the solution of the heat equation (governing term) is perturbed by the telegraph distribution (which represents the impact of the fractional derivative).

Moreover, when $u$ is $G_{1}^{\frac{1}{2}, 1}$, it can be understood as the distribution of a particle moving back and forth the real line with velocities $\pm c$ for a random time interval. Making $\alpha=\frac{1}{2}$ in (53) we get the following series representation of $G_{1}^{\frac{1}{2}, 1}$

$$
G_{1}^{\frac{1}{2}, 1}(x, t)=\delta(x)+\frac{1}{2 c \sqrt{t}} \sum_{k=0}^{+\infty} \frac{(-2 \lambda \sqrt{t})^{k}}{k !} \sum_{p=0}^{+\infty} \frac{\left(-\frac{p+1}{2}\right)_{k}}{\Gamma\left(\frac{1+k-p}{2}\right)}\left(-\frac{|x|}{c \sqrt{t}}\right)^{p} .
$$

\section{Graphical representations of $G_{n}^{\beta}$}

In this section we present and discuss some plots of $G_{n}^{\alpha, \beta}$, for $a, c=1, n=1,2,3$ and some values of the fractional parameters $\alpha$ and $\beta$. The plots were made summing the alternate series using the MatLab code ALTSUM [14], which provides a convergence acceleration of alternating series.

\subsection{Case $n=1$}

Putting $n=1, a=c=1$ in (40) we obtain

$$
G_{1}^{\alpha, \beta}(x, t)=\delta(x)-\frac{1}{2 t^{\frac{\beta}{2}}} \sum_{k=0}^{+\infty} \frac{\left(-t^{\beta-\alpha}\right)^{k}}{k !} \sum_{p=0}^{+\infty} \frac{\left(\frac{p+1}{2}\right)\left(1-\frac{p+1}{2}\right)_{k-1}}{\Gamma\left(1-\frac{\beta(p+1)}{2}+(\beta-\alpha) k\right) p !}\left(-\frac{|x|}{t^{\frac{\beta}{2}}}\right)^{p} .
$$

We show in Figure 1, the graphical representation of the reduced Green function $G_{1}^{\alpha, \beta}(x, 1)$ for some values of the fractional parameters $\alpha$ and $\beta$. 

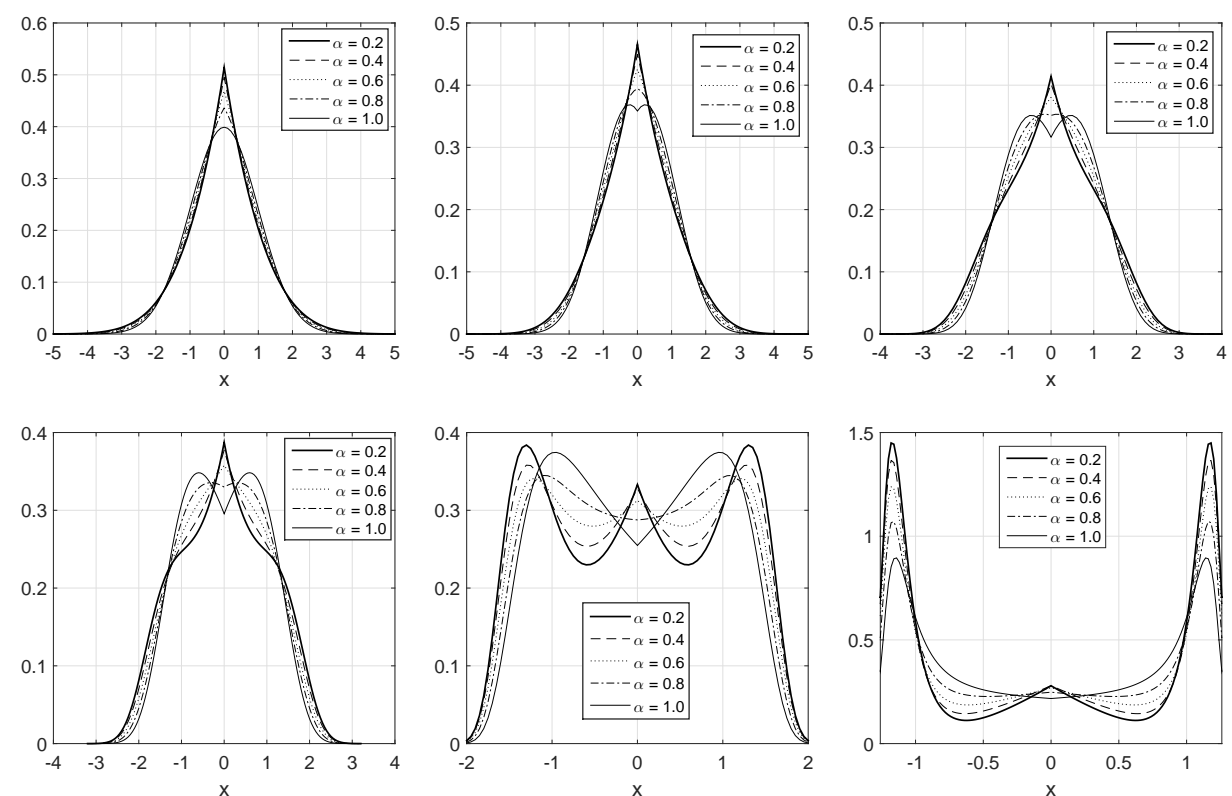

Figure 1: Plots of $G_{1}^{\alpha, \beta}(x, 1)$ for $\alpha=0.2,0.4,0.6,0.8,1.0, \beta=1.0,1.2,1.4$ (1st line, from left to right), and $\beta=1.5,1.7,1.9$ (2nd line, from left to right).

When $t=1$ and $n=1$ our FS corresponds to a fast perturbed wave phenomena (the perturbation is due to the fractional parameter $\alpha$ ), in fact, these plots are deformations of those presented in Section 7.1 of [8]. From the analysis of the plots we conclude that the first derivative of our functions has a discontinuity in $x=0$. We can also derive that for small values of $\beta \in] 1,2]$ the FS has only one extreme point in $x=0$, and then the FS starts to have two symmetric maximum points that move apart from the origin. Moreover, the plots shrink horizontally as $\beta \rightarrow 2^{-}$. The influence of the parameter $\alpha$ can also be seen in the absolute value of the two symmetric maximum points, since the increase of the parameter $\alpha$ results in a increase of the absolute value of the maxima points.

In Figures 2, 3, and 4 we show the time evolution of $G_{1}^{\alpha, \beta}(x, t)$, for $a, c=1$ and some values of $\alpha$ and $\beta$.
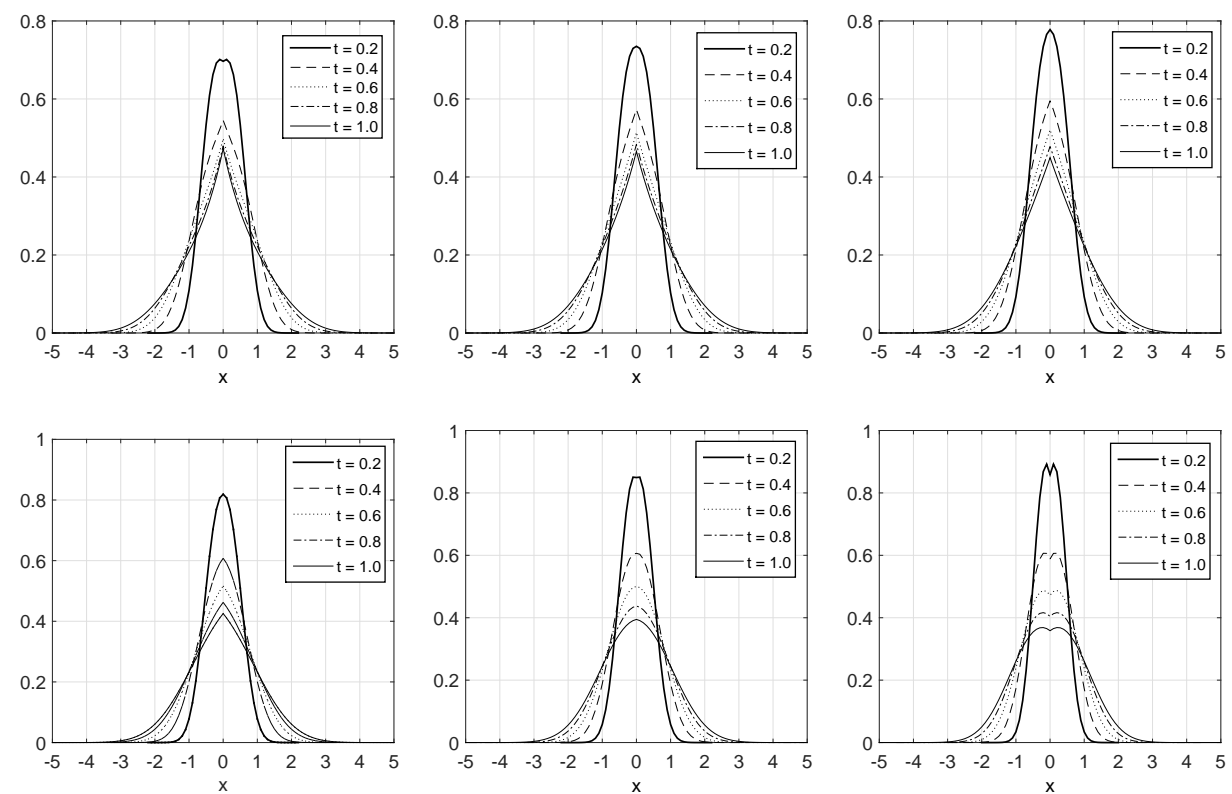

Figure 2: Plots of $G_{1}^{\alpha, \beta}$ for $\beta=1.2, t=0.2,0.4,0.6,0.8,1.0$, and $\alpha=0,0.2,0.4$ (1st line, from left to right), $\alpha=0.6,0.8,1.0$ (2nd line, from left to right). 

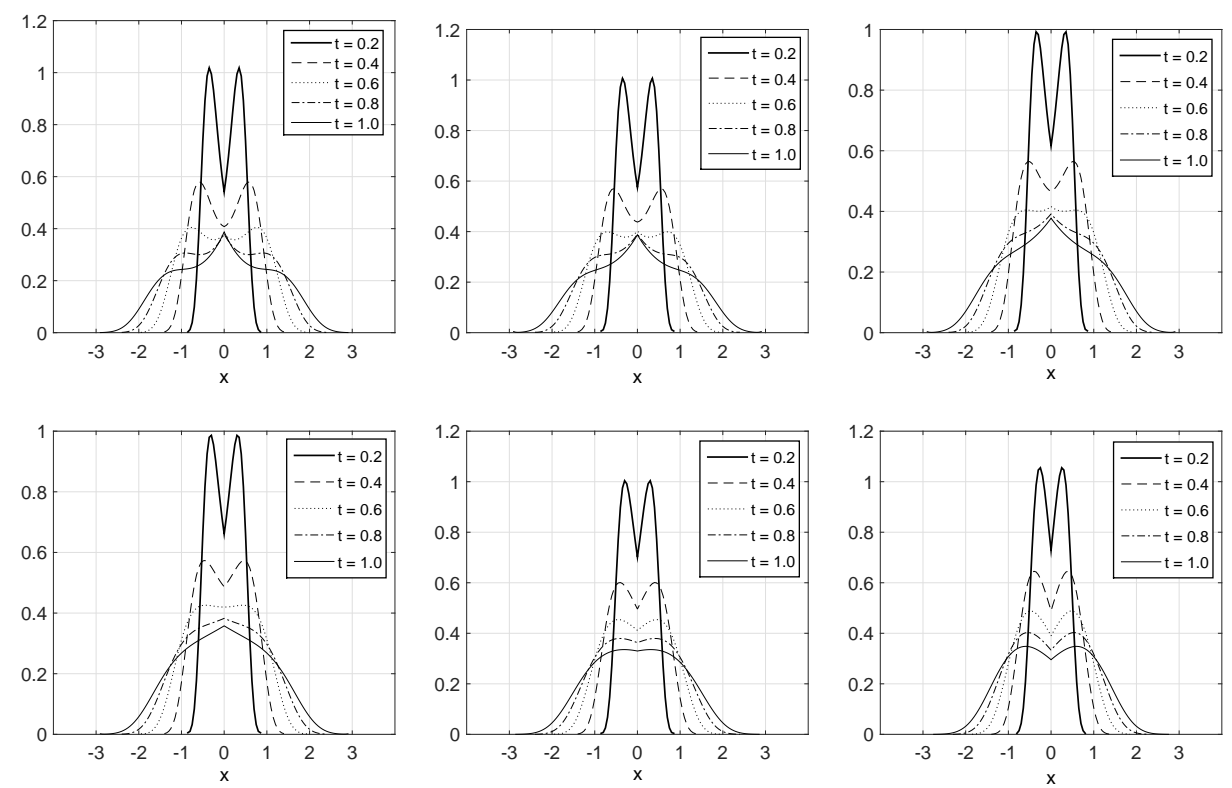

Figure 3: Plots of $G_{1}^{\alpha, \beta}$ for $\beta=1.5, t=0.2,0.4,0.6,0.8,1.0$, and $\alpha=0,0.2,0.4$ (1st line, from left to right), $\alpha=0.6,0.8,1.0$ (2nd line, from left to right).
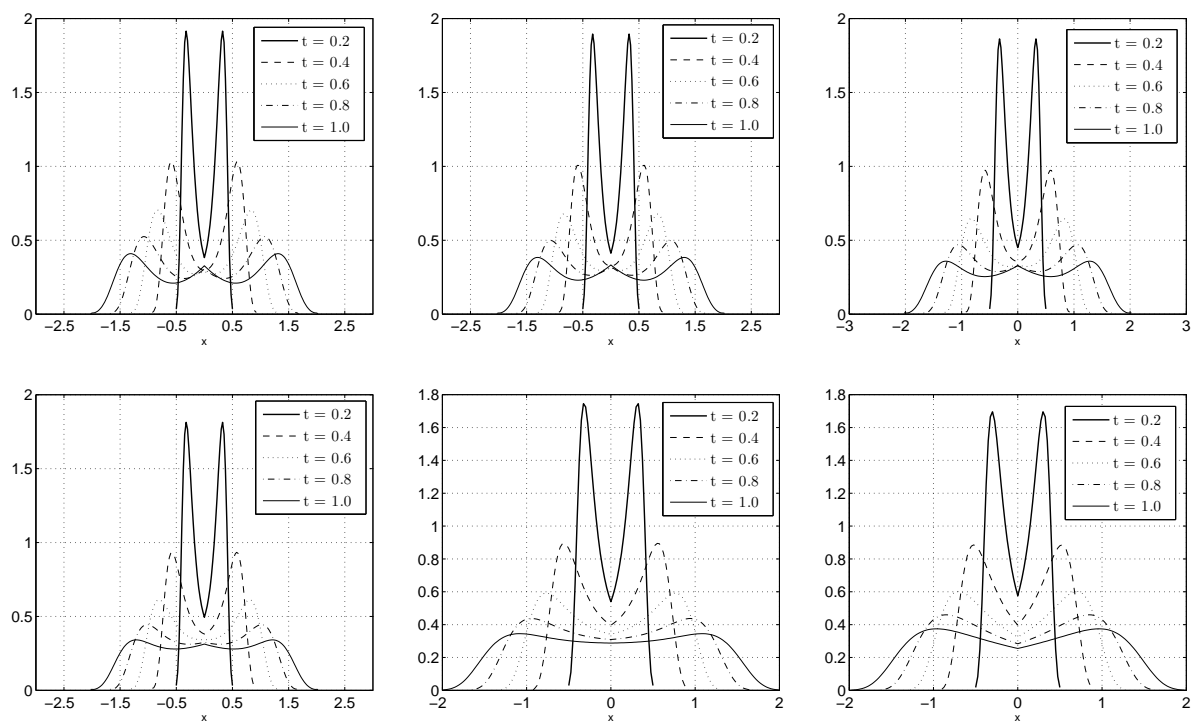

Figure 4: Plots of $G_{1}^{\alpha, \beta}$ for $\beta=1.7, t=0.2,0.4,0.6,0.8,1.0$, and $\alpha=0,0.2,0.4$ (1st line, from left to right), $\alpha=0.6,0.8,1.0$ (2nd line, from left to right).

From Figures 2, 3 and 4 it is readily seen that the with the increasing of time the behavior of the FS changes in the origin and the decay becomes slower. 


\subsection{Case $n=2$}

Putting $n=2, a=c=1$ in (43) we obtain

$$
\begin{aligned}
G_{2}^{\alpha, \beta}(x, t)= & \delta(x)+\frac{1}{4 c^{2} \pi t^{\beta}} \sum_{i=0}^{+\infty} \frac{B_{1}}{i !(i+1) ! \Gamma(1-\beta(1+i))}\left(\frac{|x|^{2}}{4 t^{\beta}}\right)^{i} \\
& -\frac{1}{4 \pi t^{\beta}} \sum_{k=1}^{+\infty} \frac{\left(-t^{\beta-\alpha}\right)^{k}}{k !} \sum_{j=0}^{k-2} \frac{(-1)^{j}}{j !} \frac{\Gamma(-j+k-1)(j+1)}{\Gamma(1-\beta(j-k-1)-\alpha k)}\left(\frac{|x|^{2}}{4 t^{\beta}}\right)^{j} \\
& +\frac{1}{\pi|x|^{2}} \sum_{k=1}^{+\infty} \frac{\left(-t^{\beta-\alpha}\right)}{k !} \sum_{j=0}^{+\infty} \frac{B_{2}}{j !(j+k-1) ! \Gamma(1-\beta j-\alpha k)}\left(\frac{|x|^{2}}{4 t^{\beta}}\right)^{j+k},
\end{aligned}
$$

where $B_{1}$ and $B_{2}$ are the following expressions

$$
\begin{aligned}
& B_{1}=-1+(1+i)\left(\ln \left(\frac{4 t^{\beta}}{|x|^{2}}\right)+\psi(2+i)+\psi(1+i)-\beta \psi(1-\beta(1+i))\right), \\
& B_{2}=-1+(j+k)\left(\ln \left(\frac{4 t^{\beta}}{|x|^{2}}\right)+\psi(j+k)+\psi(1+j)-\beta \psi(1-\beta j-\alpha k)\right) .
\end{aligned}
$$

In Figure 5 we present some plots of $G_{2}^{\alpha, \beta}$.
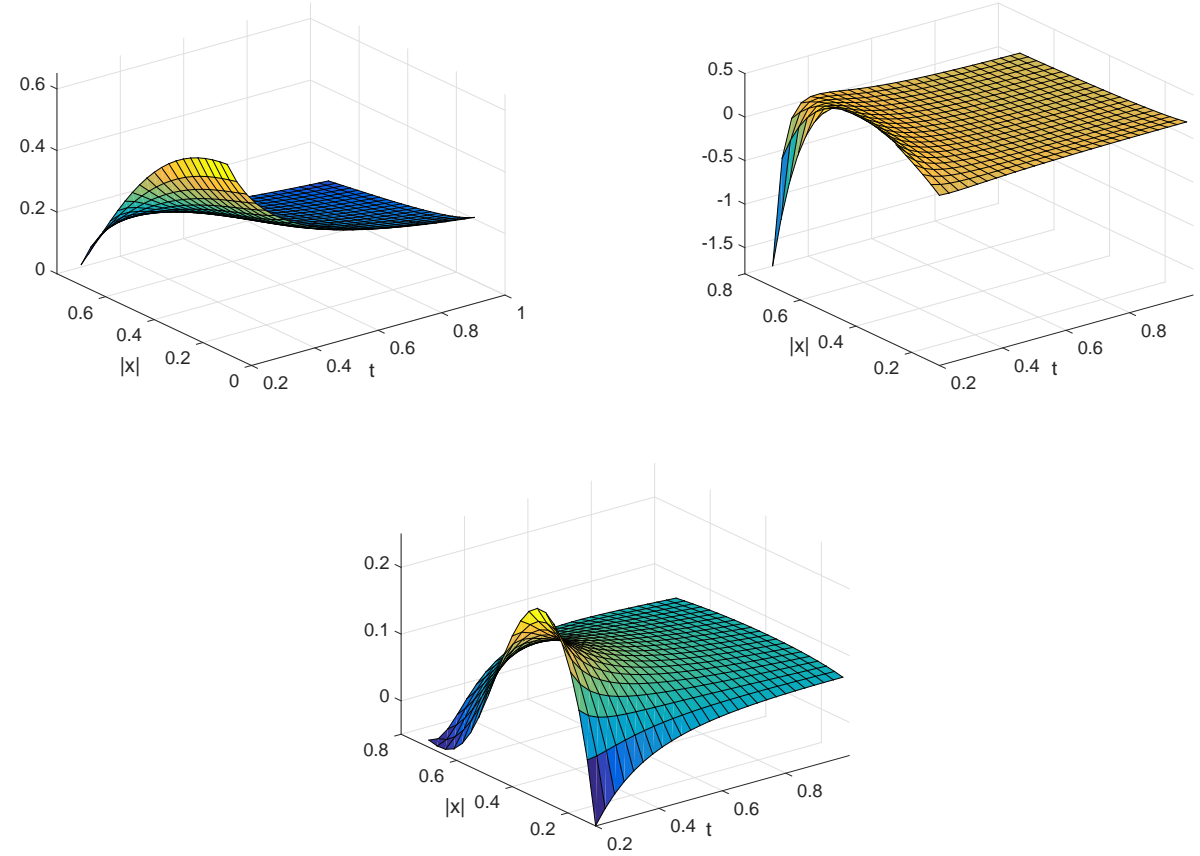

Figure 5: Plots of $G_{2}^{\alpha, \beta}$ for $\beta=1.2, \alpha=0.6$ (left), $\beta=1.4, \alpha=0.4$ (center), and $\beta=1.5, \alpha=0.8$ (right).

From Figure 5, we see that the behaviour of the FS changes in time and in space accordingly with the parameters $\alpha$ and $\beta$ chosen.

\subsection{Case $n=3$}

Putting $n=3, a=c=1$ in (40) we obtain

$$
\begin{aligned}
G_{3}^{\alpha, \beta}(x, t)= & \delta(x)+\frac{1}{4 \pi|x|}\left(\frac{1}{\Gamma(1-\beta) t^{\beta}}+\frac{t^{-\alpha}}{\Gamma(1-\alpha)}\right) \\
& +\frac{1}{\pi\left(4 t^{\beta}\right)^{\frac{3}{2}}} \sum_{k=0}^{+\infty} \frac{\left(-t^{\beta-\alpha}\right)^{k}}{k !} \sum_{p=0}^{+\infty} \frac{(p+3)\left(1-\frac{p+3}{2}\right)_{k-1}}{(p+1) \Gamma\left(1-(\beta-\alpha) k-\frac{\beta(p+3)}{2}\right) p !}\left(-\frac{|x|}{t^{\frac{\beta}{2}}}\right)^{p}
\end{aligned}
$$

We show in Figure 6 the graphical representation of $G_{3}^{\alpha, \beta}$. 

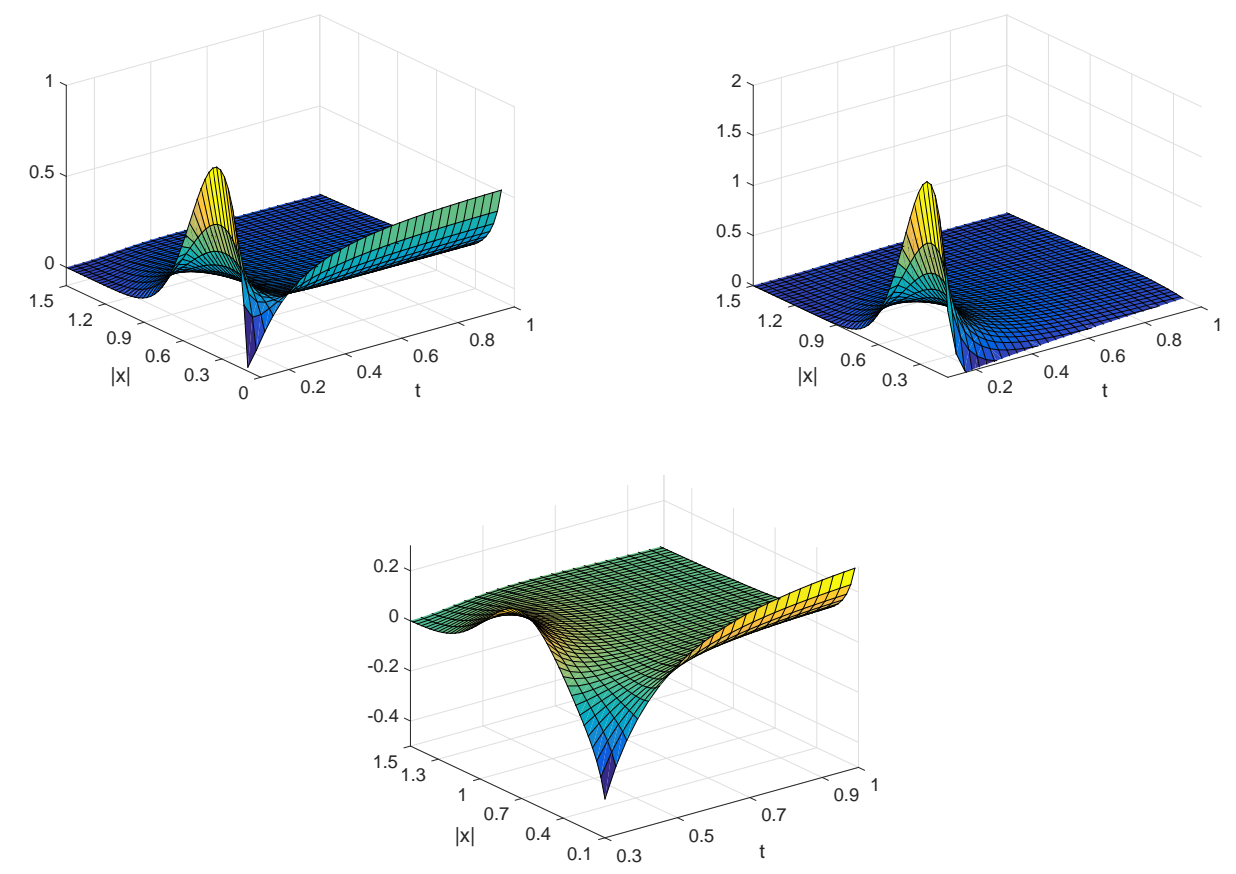

Figure 6: Plots of $G_{3}^{\alpha, \beta}$ for $\beta=1.2, \alpha=0.2$ (left), $\beta=1.2, \alpha=1.0$ (center), and $\beta=1.4, \alpha=0.4$ (right).

For the three dimensional case we observe similar behaviours as in the case $n=1$, with the difference that these solutions are no longer non-negative. We also observe a different range of values of $G_{3}^{\alpha, \beta}$, which is caused by different choices of the parameters $\alpha$ and $\beta$ in the plots.

Acknowledgement: The authors were supported by Portuguese funds through the CIDMA - Center for Research and Development in Mathematics and Applications, and the Portuguese Foundation for Science and Technology ("FCT-Fundaçâo para a Ciência e a Tecnologia"), within project UID/MAT/ 0416/2013. N. Vieira is Auxiliar Researcher, under the FCT Researcher Program 2014 (Ref: IF/00271/2014).

\section{References}

[1] M. Abramowitz and I.A. Stegun, Handbook of mathematical functions with formulas, graphs, and mathematical tables. 10th printing, National Bureau of Standards, Wiley-Interscience Publication, John Wiley \& Sons, New York etc., 1972.

[2] R.G. Buschman, H-functions of two variables III, Pure Appl. Math. Sci., 9, (1978), 13-18.

[3] R.G. Buschman, H-functions of two variables I, Indian J. Math., 20, (1978), 132-153.

[4] R.F. Camargo, A.O. Chiacchio and E.C. de Oliveira, Differentiation to fractional orders and the fractional telegraph equation, J. Math. Phys., 49-No.3, (2008), Article ID 033505, 12p.

[5] R.C. Cascaval, E.C. Eckstein, L.C. Frota and J.A. Goldstein, Fractional telegraph equations, J. Math. Anal. Appl., 276-No.1, (2002), 145-159.

[6] J. Chen, F. Liu, V. Anh, Analytical solution for the time-fractional telegraph equation by the method of separating variables, J. Math. Anal. Appl., 338-No.2, (2008), 1364-1377.

[7] A. Erdélyi, W. Magnus, F. Oberhettinger and G. Tricomi, Tables of integral transforms-Vol.II, Bateman Manuscript Project, California Institute of Technology, McGraw-Hill Book Company, New York-Toronto-London, 1954.

[8] M. Ferreira and N. Vieira, Fundamental solutions of the time fractional diffusion-wave and parabolic Dirac operators, J. Math. Anal. Appl., 447-No.1, (2017), 329-353.

[9] R. Gorenflo, A.A. Kilbas, F. Mainardi and S. Rogosin, Mittag-Leffler functions. Theory and applications, Springer Monographs in Mathematics, Springer, Berlin, 2014.

[10] H.J. Haubold, A.M. Mathai and R.K. Saxena, Mittag-Leffler functions and their applications, J. Appl. Math., (2011), Article ID 298628, 51 p. 
[11] A. Kilbas, H.M. Srivastava and J.J. Trujillo, Theory and applications of fractional differential equations, NorthHolland Mathematics Studies-Vol.204, Elsevier, Amsterdam, 2006.

[12] A. Kilbas and M. Saigo, H-transforms. Theory and applications, Analytical Methods and Special Functions-Vol.9, Chapman \& Hall/CRC, Boca Raton, FL, 2004.

[13] Y. Luchko and R. Gorenflo, An operational method for solving fractional differential equations with the Caputo derivatives, Acta Math. Vietnam., 24-No.2, (1999), 207-233.

[14] J. Lundgren, Convergence acceleration of alternating series, (https://www.mathworks.com/matlabcentral/ fileexchange/25200-altsum), MATLAB Central File Exchange, 2011. Access: 12/09/2016.

[15] M.O. Mamchuev, Solutions of the main boundary value problems for the time-fractional telegraph equation by the Green function method, Fract. Calc. Appl. Anal., 20-No.1, (2017), 190-211.

[16] E. Orsingher and L. Beghin, Time-fractional telegraph equations and telegraph processes with Brownian time, Probab. Theory Relat. Fields, 128-No.1, (2004), 141-160.

[17] I. Podlubny, Fractional Differential Equations. An introduction to fractional derivatives, fractional differential equations, to methods of their solution and some of their applications, Mathematics in Science and Engineering 198, Academic Press, San Diego, 1999.

[18] S.G. Samko, A.A. Kilbas and O.I. Marichev, Fractional integrals and derivatives: theory and applications, Gordon and Breach, New York, NY, 1993.

[19] S. Yakubovich and M.M. Rodrigues, Fundamental solutions of the fractional two-parameter telegraph equation, Integral Transforms Spec. Funct., 23-No.7, (2012), 509-519. 